\title{
RACSAMI
}

Rev. R. Acad. Cien. Serie A. Mat.

VOL. 102 (1), 2008, pp. 39,73

Geometría y Topología / Geometry and Topology

Artículo panorámico / Survey

\section{Inverse Limits, Economics, and Backward Dynamics}

\author{
Judy Kennedy
}

\begin{abstract}
We survey recent papers on the problem of backward dynamics in economics, providing along the way a glimpse at the economics perspective, a discussion of the economic models and mathematical tools involved, and a list of applicable literature in both mathematics and economics.
\end{abstract}

\section{Límites inversos, Economía y Dinámica Regresiva}

Resumen. Examinamos artículos recientes sobre el problema de la dinámica regresiva en Economía, haciendo una breve incursión en la perspectiva económica, y presentando una discusión de los modelos económicos y herramientas matemáticas involucrados, y una relación de literatura pertinente en Matemáticas y en Economía.

\section{Introduction}

Our focus here is on introducing some problems from economics to mathematicians, showing how mathematical problems arise from these economic problems, and then showing how tools from inverse limits, topology, dynamics, and measure theory shed some light on the solution of the problems. There is no claim that this survey is exhaustive; rather our goal is to describe how inverse limits have recently been applied to the problem of "backward" dynamics in economics. We begin with a brief discussion of the goals of economics, a glimpse at the economic perspective, and a list of some of the economics terms encountered in our investigation of several models. Then we discuss the particular models we have studied, the results obtained, and what it all means for economics. We assume the reader has more familiarity with the mathematical tools involved than with modern economics. However, extensive references are given at the end from both the economics and mathematics literature.

\section{A Glimpse at Economics}

Let's start with a familiar example from economics that we all see in a first semester calculus course: If $C(x)$, the cost function, is the cost of producing $x$ units of a certain product, then the marginal cost is the rate of change of $C$ with respect to $x$. That is, the marginal cost function is the derivative $C^{\prime}(x)$ of the cost function. The average cost function $c(x)=\frac{C(x)}{x}$ represents the cost per unit when $x$ units are produced.

Presentado por Pedro Jiménez Guerra.

Recibido: 27 de septiembre de 2007. Aceptado: 5 de diciembre de 2007.

Palabras clave / Keywords: inverse limits, applications to economics, backward dynamics, indecomposable continua, Knaster bucket handle, chaos, entropy, measure, natural invariant measure, unimodal, chainable, invariant measure, simple dynamics, logistic maps, tent maps, cash-in-advance model, overlapping generations model, period three, difference equation.

Mathematics Subject Classifications: primary 54H20, 39A05, 37N99; secondary 37N40, 37B45, 37E05, 54F15

(C) 2008 Real Academia de Ciencias, España. 


\section{J. Kennedy}

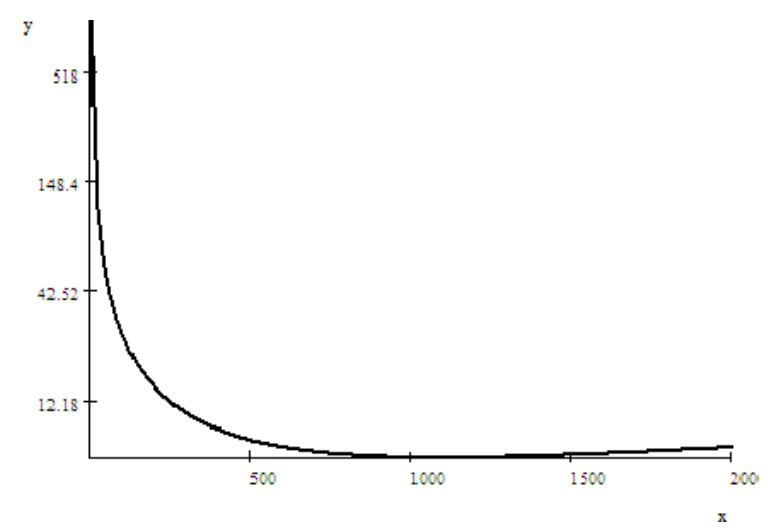

Figure 1. The average cost function

An example: A company estimates the cost (in dollars) of producing $x$ items is $C(x)=2400+2 x+$ $0.002 x^{2}$. The average cost function is then $c(x)=\frac{C(x)}{x}=2400 / x+2+0.002 x$, and the marginal cost function is $C^{\prime}(x)=2+0.004 x$. If we graph the average cost function, we get Figure 1

It appears that the average cost function has an absolute minimum, and we can find this minimum by differentiating $c(x)$ and setting the derivative to $0: c^{\prime}(x)=-2400 x^{-2}+0.002=0$, which gives $x$ approximately 1095.4. Since it is not possible to produce 0.4 units, it would appear that producing 1095 units would give us a minimum average cost. We can check this by taking the second derivative of $c(x)$ : $c^{\prime \prime}(x)=4800 / x^{3}$. Thus, the second derivative is positive for $x$ positive, so the function is concave up for $x$ positive, and since $x$ must be positive to make sense, this means that producing 1095 units should give us the minimum average cost possible.

This little example gives a glance at the mathematical tools economists use to make a decision as to how best proceed. The model here is simple in that the cost function is a differentiable function of only one variable. The example is a problem from microeconomics, rather than macroeconomics, and it is also an example of an optimization problem. Microeconomics is used to describe the decision making processes of individuals and firms, while macroeconomics applies to the study of relations between broad economic aggregates. Not surprisingly, optimization is fundamental in solving many problems in economics.

In economics, utility is a measure of the relative satisfaction or desiredness derived from the consumption of goods. The idea is that one attempts to increase one's utility. Economists actually sometimes call this one's happiness. A good is an object (a physical or tangible product) or service that increases one's utility, directly or indirectly. (Yes, this is circular, as utility is defined in terms of goods and vice versa.) Goods are usually modelled as having decreasing marginal utility. For example, the first car one purchases is more useful than the fourth (especially when these purchases mean that four cars are now owned). Goods are normal if demand for them increases when income increases. The term normal good does not necessarily refer to the quality of the good. An agent is an actor in a model that (generally) solves an optimization problem. More recently, it has come to be interpreted more broadly as a persistent individual, social, biological, or physical entity interacting with other such entities within the context of a dynamic multi-agent system. An endowment is the amount of something that a person, country, etc., simply has, rather than their having to somehow acquire it. Consumption refers to the final use of goods and services to provide utility. The market clearing condition holds when supply equals demand in a model. Perfect foresight is said to hold when an agent in a model has exact knowledge of the future (relative to the model). In an economics model exogenous refers to an action or object coming from outside the system. An exogenous change is one that comes from outside the model and is not explained by the model. On the other hand, endogenous refers to something generated within the model and explained by the model.

Originally, an equilibrium in economics meant simply a state of the world where economic forces are balanced and in the absence of external influences the equilibrium values of economic variables will 
not change. For example, in a free market and simple supply-demand model where the demand curve is decreasing, the supply curve is increasing, and the curves intersect only at one point, an equilibrium occurs at the price where the curves intersect. When the price is above the equilibrium point there is a surplus of supply, and when the price is below the equilibrium point there is a shortage of supply. Excess supply would lead to price cuts, and would lead to a decrease in supply (since there would be less incentive to produce and sell the product), leading to an excess demand, which would then lead to price increases, thus abolishing the surplus, and leading to an increase in supply, and the whole cycle starts again. In theory, over time, this disequilibrium state (price too high, price too low) would tend to disappear, as the supply-demand forces try to balance themselves.

Economists define models that represent the choices made by agents. The mathematics is representative of the trade-offs inherent in decision making, and provides (hopefully) tractable methods for finding optimal behavior. It also enables the derivation of testable hypotheses that should hold if the model accurately reflects behavior. The universal assumption is that agents make choices that maximize returns (benefits). If the set of feasible choices is unlimited, then unconstrained maximization is involved. If the set of choices is limited by, say budgetary constraints, resource scarcity, or legal barriers, then constrained maximization is involved.

As in setting up any model, the first step is to specify the variables and the parameters. The parameters are constants in relation to the variables of the model, but in comparative studies (static or dynamic) we can see what happens to the outcome when one or more parameters change. The next step is to formulate the conditions we lay down for the operation of the model. These conditions are of three kinds:

Definitions and identities. These specify a relation that holds by definition. For example, total demand could be defined as the sum of consumption and investment demand.

Functional relations. These are dependences assumed in the model. For example, we might assume that production in period $t, P_{t}=f\left(C_{t}, L_{t}\right)$, where $C_{t}$ denotes capital in period $t$, and $L_{t}$ denotes labor in period $t$.

Conditions of the model. These come from assumptions about the equilibrium or disequilibrium operation of the model. Such an assumption in the CIA model discussed below is that household may not use the lump-sum transfer $\theta M_{t}$ from the government at period $t$ to purchase cash goods $\left(c_{1 t}\right)$ during that same period.

Finally, the conditions of the model must be reduced as much as possible to make the model easier to study. The final formulation of the model may be in either continuous or discrete form. In a discrete analysis, the flow of time is divided into successive periods of constant length, which is taken as the time unit. If the model is dynamic, in the sense of involving variables at different time periods, the result is a difference equation (or perhaps a system of difference equations). In a continuous analysis with a dynamic model, the result is a differential equation (or system of differential equations). The models we discuss here are dynamic, and involve a discrete analysis.

The simple example above involved unconstrained maximization (or rather unconstrained minimization) of a function $f(x)$ of a single variable. In the example, average cost was being minimized. The function $f(x)$ could also, say, represent profits for a given quantity of $x$ produced, or it could represent utility, with $x$ being the quantity consumed. If $f(x)$ represented profits, then the object would be to determine what quantity $x$ to choose to maximize profits. If $f(x)$ represents utility, the object would be to determine how much of $x$ to consume to maximize utility.

Models are rarely as simple as the one discussed above. They may well be multidimensional, and require the tools of optimization theory for solution. Such a multidimensional problem could arise, if, say, profits are a function $g$ of capital, labor, and energy; or if utility $h$ is a function of food, water, and leisure. If constrained maximization is involved, a tool widely used by economists is the Lagrange multiplier.

A significant portion of the modern literature in economics is quite mathematical in nature. Indeed, many papers are essentially mathematics papers with a theorem-proof-example format. (The Michener- 
Ravikumar paper [66] is an excellent example of such a paper.) Economists use tools from analysis, differential equations, partial differential equations, and dynamical systems as needed. With the advent of powerful computers, it has become possible to study ever more complicated models, so modern computation also plays an important role.

The dynamical systems resulting from dynamic models need not be differentiable, or even continuous; indeed, they may not even be well defined functions! In this case, the usefulness of the usual tools from calculus is somewhat limited, as we shall see. In fact, we focus here on several such models. The cash-inadvance model and the overlapping generations model share the problem of "backward dynamics". These two models are represented by functions that are not well defined forward in time. We also briefly discuss the Christiano-Harrison model, which is not a function backward or forward in time. All three models come from macroeconomics. The cash-in-advance model and overlapping generations model, in particular, would be encountered in an introductory graduate text on macroeconomics.

For the discussion that follows, references to the economics literature are [3, 12, 15, 22, 24, 27, 28, 29] (30, 31, 33, 36, 48, 51, 53, 55, 56, 58, 61, 62, 63, 65, 66, 74, $76,78,81,82]$. References to the mathematics literature are [1, 2, 4, 5, 5, 6, 7, 8, 9, 10, 13, 14, 16, 17, 18, 19, 20, 21, 23, 25, 26, 32, 34, 35, 37, 38, 39, 40

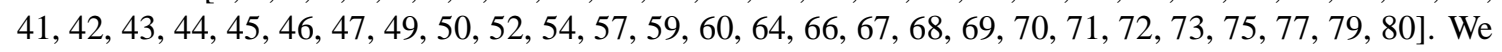
would like to thank David Stockman for his help and advice, and we would also like to thank Wikipedia.

\section{The Cash-in-advance model}

Cash-in-advance models are used to model monetary phenomena. Here we consider a particular cashin-advance model. The model is the standard endowment CIA model of Lucas and Stokey [58], and we closely follow the exposition of R. Michener and B. Ravikumar [66]. The model gives rise to an implicitly defined difference equation that has the unusual property that it is well-determined backward in time, but not forward in time. We sketch the derivation of the model from the economics assumptions made. For more details, see [66]. Note that, even in this quite idealized model, the process of going from the economic assumptions to a simplified mathematical model takes a fair amount of work.

- In the model, households are choosing how much cash to hold over time.

- Households are representative, i.e., they are all "typical" households and behave the same way.

- Holding cash today allows the household to purchase certain goods and services which we will call cash goods.

- Other goods and services can be bought on credit and do not require cash which we will call credit goods.

- The implicit cost of holding cash is the interest income foregone if the household had instead held the cash in the form of another asset, say bonds. The choice to hold cash involves a trade-off: the benefit of being able to purchase cash goods and services against the cost of the foregone interest income. It is assumed that the household makes this trade-off optimally, and so the household's problem is best set in the framework of a dynamic optimization problem.

- Consumption in the future is worth less to the household so the benefit from future consumption is discounted. Accordingly, there is a parameter $\beta$, called the discount factor, which is strictly between 0 and 1 , and which quantifies exactly how future consumption is discounted.

- To purchase the cash good $c_{1 t}$ at time $t$ the household must have cash $m_{t}$. This cash is carried forward from time $t-1$ and in this sense the household is required to have cash in advance of purchasing the cash good.

- The credit good $c_{2 t}$ does not require cash, but can be bought on credit. 
- The household has an endowment $y$ each period that can be transformed into the cash and credit goods.

- In equilibrium, all of the endowment $y$ is transformed into cash and credit goods so that the total of cash goods and credit goods is $y$, i.e., $c_{1 t}+c_{2 t}=y$.

- In equilibrium, this allows the cash good to be substituted for the credit good one-for-one, so both goods must sell for the same price $p_{t}$ and the endowment must be worth this price per unit as well.

- The government in this economy transfers money to each household at each period with $M_{t}$ denoting the total money holding of the household at each time $t$, and the government giving the household the amount $\theta M_{t}$, where $\theta$ is another real-valued parameter. (Furthermore, $\theta$ is a parameter that the government can control.)

- The sequence $\left\{M_{t}\right\}_{t=0}^{\infty}$ is called the money supply.

Both $U$ and $W$ represent utility functions: $U$ denotes the utility gained from one choice of amount of cash good and credit good, while $W$ represents the total utility gained from an entire sequence of cash goods and credit goods purchased over time. More specifically, the household has preferences over sequences of the cash good $\left(c_{1 t}\right)$ and credit good $\left(c_{2 t}\right)$ represented by a utility function (real-valued) of the form

$$
W\left(\left\{c_{1 t}, c_{2 t}\right\}_{t=0}^{\infty}\right):=\sum_{t=0}^{\infty} \beta^{t} U\left(c_{1 t}, c_{2 t}\right) .
$$

One sequence $\left\{c_{1 t}, c_{2 t}\right\}_{t=0}^{\infty}$ is preferred over another sequence $\left\{\tilde{c}_{1 t}, \tilde{c}_{2 t}\right\}_{t=0}^{\infty}$ if and only if

$$
W\left(\left\{c_{1 t}, c_{2 t}\right\}_{t=0}^{\infty}\right)>W\left(\left\{\tilde{c}_{1 t}, \tilde{c}_{2 t}\right\}_{t=0}^{\infty}\right) .
$$

Now consider the household's optimization problem: The household seeks to maximize $W$ by its choice of $\left\{c_{1 t}, c_{2 t}, m_{t+1}\right\}_{t=0}^{\infty}$ subject to the constraints $c_{1 t}, c_{2 t}, m_{t+1} \geq 0$. The cash-in-advance constraint says that the amount spent on the cash good $p_{t} c_{1 t}$ must be no more than cash on hand $m_{t}$. The budget constraint on cash holdings for next period says that the cash carried over into next period $\left(m_{t+1}\right)$ can be no greater than the income $\left(p_{t} y\right)$ plus cash not spent $\left(m_{t}-p_{t} c_{1 t}\right)$ plus the transfer of cash from the government $\left(\theta M_{t}\right)$ minus the amount spent on the credit good $\left(p_{t} c_{2 t}\right)$. These two constraints translate into inequalities (2) and (3) below. Thus,

$$
\begin{aligned}
p_{t} c_{1 t} & \leq m_{t}, \\
m_{t+1} & \leq p_{t} y+\left(m_{t}-p_{t} c_{1 t}\right)+\theta M_{t}-p_{t} c_{2 t},
\end{aligned}
$$

where we are taking as given $m_{0}$ and $\left\{p_{t}, M_{t}\right\}_{t=0}^{\infty}$. (The money supply $\left\{M_{t}\right\}$ follows a constant growth path $M_{t+1}=(1+\theta) M_{t}$ where $\theta$ is the growth rate and $M_{0}>0$ given.)

In [66] the authors make assumptions on the function $U$ so that the solution to this problem will be interior and the solution to the first-order conditions and transversality condition will be necessary and sufficient. (First order conditions refer to the first derivative conditions. Transversality conditions are a bit more mysterious. Transversality conditions are terminal conditions and are necessary for optimization. Since our model is an infinite horizon one, for us it is a condition on the tail of the sequence chosen, and corresponds to condition (7) below.)

Assumption 1[66 p. 1120]: The function $U: \mathbb{R}_{+}^{2} \rightarrow \mathbb{R}$ is increasing in both arguments, strictly concave, and $\mathcal{C}^{2}$. Both $c_{1 t}$ and $c_{2 t}$ are assumed to be normal goods. Further, to guarantee interior solutions we will assume

$$
\lim _{c \rightarrow 0} U_{1}(c, y-c)=\lim _{c \rightarrow y} U_{2}(c, y-c)=\infty,
$$

and that $U_{1}(y, 0)<\infty$ and $U_{2}(0, y)<\infty$. 
The assumption that $U$ is increasing in both arguments embodies the notion that more is preferred to less. Strict concavity implies (among other things) that $U_{11}<0$ and $U_{22}<0$, which represents what economists call diminishing marginal utility. The extra enjoyment from more of each good is positive, but diminishes as more of the good is consumed. Being a normal good simply means that, all else equal, if the household has more income, more of the good will be consumed. The other assumptions on the partial derivatives imply that if feasible, the household will choose $0<c_{1}<y$ and $0<c_{2}<y$, i.e., the solution will be interior. In the economics literature, these assumptions are fairly standard.

To solve the household's constrained optimization problem the Lagrangian method is used in [66]:

$$
\mathcal{L}=\sum_{t=0}^{\infty} \beta^{t}\left\{U\left(c_{1 t}, c_{2 t}\right)+\mu_{t}\left(m_{t}-p_{t} c_{1 t}\right)+\lambda_{t}\left[p_{t}\left(y-c_{2} t\right)+\left(m_{t}-p_{t} c_{1 t}\right)-m_{t+1}+\theta M_{t}\right]\right\}
$$

where $\left\{\mu_{t}, \lambda_{t}\right\}$ are non-negative Lagrange multipliers. The first-order conditions and transversality condition for this problem are

$$
\begin{aligned}
U_{1}\left(c_{1 t}, c_{2 t}\right) & =p_{t}\left(\lambda_{t}+\mu_{t}\right), \\
U_{2}\left(c_{1 t}, c_{2 t}\right) & =p_{t} \lambda_{t}, \\
\lambda_{t} & =\beta\left(\lambda_{t+1}+\mu_{t+1}\right), \\
0 & =\lim _{t \rightarrow \infty} \beta^{t} \lambda_{t} m_{t+1} .
\end{aligned}
$$

Equations (4), (5) and (6) come from applying the method of Lagrange multipliers. Upon substituting equations (4) and (5) into (6), we get

$$
U_{2}\left(c_{1 t}, c_{2 t}\right) / p_{t}=\beta U_{1}\left(c_{1 t+1}, c_{2 t+1}\right) / p_{t+1} .
$$

This condition reflects that at the optimum, the household must be indifferent between spending a little more on the credit good (giving a marginal benefit $U_{2}\left(c_{1 t}, c_{2 t}\right) / p_{t}$ ) versus savings the money and purchasing the cash good in the next period (giving a marginal benefit $\left.\beta U_{2}\left(c_{1 t+1}, c_{2 t+1}\right) / p_{t+1}\right)$.

An equilibrium in the model is essentially a sequence of prices such that supply equals demand. More formally, an equilibrium is by definition a collection of sequences $\left\{c_{1 t}, c_{2 t}, m_{t}, M_{t}, p_{t}\right\}_{t=0}^{\infty}$ such that, $M_{t+1}=(1+\theta) M_{t}$ (the money supply follows the stated policy rule), $m_{t}=M_{t}$ (demand for money equals the supply of money) and $c_{1 t}+c_{2 t}=y$ (demand for goods equals the supply of goods), and the solution to the household optimization problem is given by $\left\{c_{1 t}, c_{2 t}, m_{t+1}\right\}_{t=0}^{\infty}$. Let $x_{t}:=m_{t} / p_{t}$. Then using the conditions that $M_{t}=m_{t}$ and $c_{2 t}=y-c_{1 t}$, equation 8 implies

$$
x_{t} U_{2}\left(c_{1 t}, y-c_{1 t}\right)=\frac{\beta}{1+\theta} x_{t+1} U_{1}\left(c_{1 t+1}, y-c_{1 t+1}\right) .
$$

If the cash-in-advance constraint (2) holds, then $c_{1 t}=x_{t}$. If not, then the Lagrange multiplier $\mu_{t}=0$ and $c_{1 t}=c:=\arg \max _{x} U(x, y-x)$. It then follows that $c_{1 t}=\min \left[x_{t}, c\right]$ for all $t$. Using this relationship we can eliminate $c_{1 t}$ and $c_{1 t+1}$ from $(9$ to get a difference equation in $x$ alone:

$$
x_{t} U_{2}\left(\min \left[x_{t}, c\right], y-\min \left[x_{t}, c\right]\right)=\frac{\beta}{1+\theta} x_{t+1} U_{1}\left(\min \left[x_{t+1}, c\right], y-\min \left[x_{t+1}, c\right]\right)
$$

or

$$
B\left(x_{t}\right)=A\left(x_{t+1}\right)
$$

where

$$
\begin{aligned}
& B(x):=x U_{2}(\min [x, c], y-\min [x, c]), \\
& A(x):=\frac{\beta}{1+\theta} x U_{1}(\min [x, c], y-\min [x, c]) .
\end{aligned}
$$




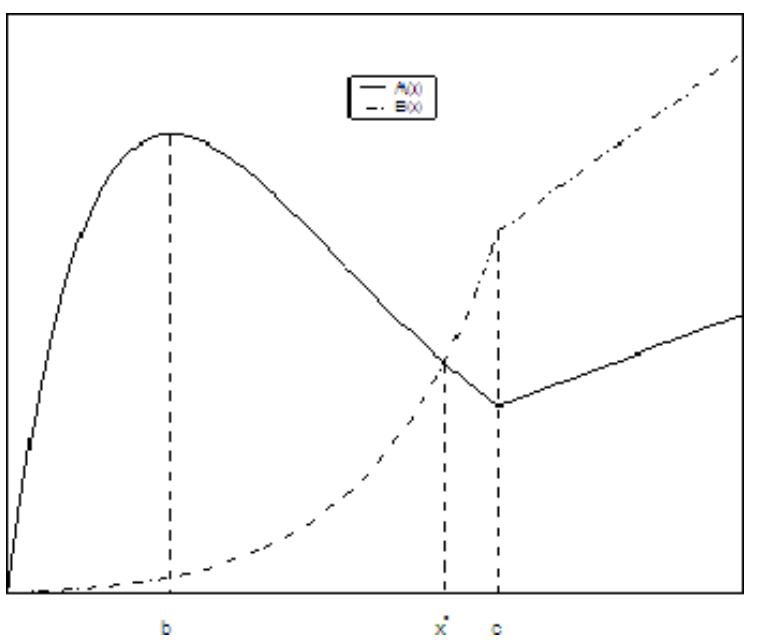

Figure 2.

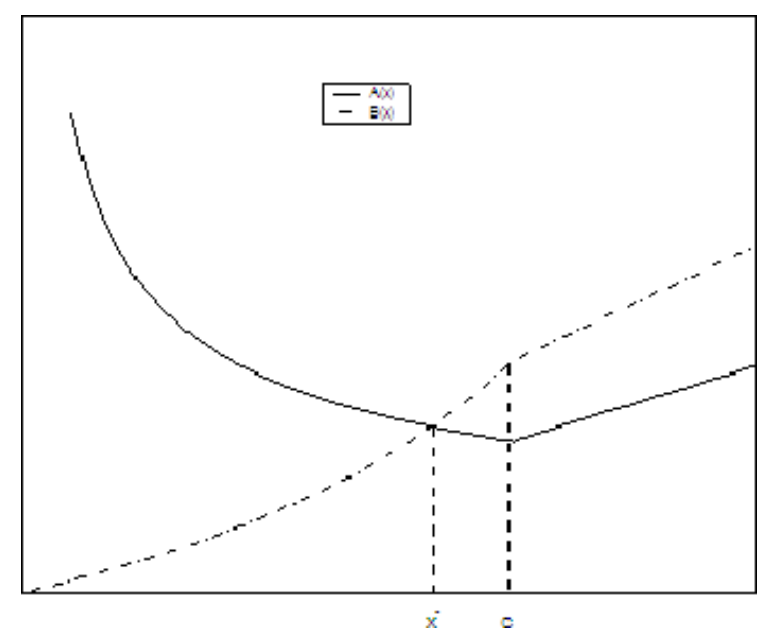

Figure 3.

See Figures 2 and 3 for illustrations of two possible configurations for $A$ and $B$. There are three possible configurations for $A$ and $B$, which are called Type I, Type II, or Type III (respectively Case I, Case II, Case III), depending on how $A$ behaves. A Type I (Case I) configuration has $A(0)=0$, $A$ increasing on $[0, b]$, decreasing on $[b, c]$, and increasing on $[c, \infty)$. A Type II (Case II) configuration has $A(0)>0, A$ increasing on $[0, b]$, decreasing on $[b, c]$, and increasing on $[c, \infty)$. A Type III (Case III) configuration has $A$ decreasing on $(0, c]$, and increasing on $[c, \infty)$. For Case III, [66] take $b=0 ; A$ may or may not be defined at 0 in this case. One can show that there is a one-to-one mapping between equilibria in the model and non-negative sequences $\left\{x_{t}\right\}$ that satisfy the difference equation (10) and transversality condition

$$
\lim _{t \rightarrow \infty} \beta^{t} U_{1}\left(\min \left[x_{t}, c\right], y-\min \left[x_{t}, c\right]\right) x_{t}=0 .
$$

Since the discount factor $\beta$ is assumed to be strictly between 0 and 1 , any solution to the difference equation (10) that is bounded from above and from below by a strictly positive constant will satisfy the transversality condition. 
Michener and Ravikumar use two more assumptions in their paper [66 p. 1125] which we give next and briefly describe what they imply for the model.

Assumption 2: There exists a $b \in[0, c)$ such that $x U_{1}(x, y-x)$ is increasing in the region $[0, b)$ and decreasing in the region $(b, c]$.

This assumption is puts additional restriction on the utility function so that the function $A(\cdot)$ is either hump-shaped or monotonically decreasing on $[0, c]$.

Assumption 3: (a) $(1+\theta)>\beta$ and (b) $b<x^{*}$.

These conditions guarantee the existence of a solution $x^{*}>0$ to $A\left(x^{*}\right)=B\left(x^{*}\right)$ and guarantee that this intersection of the two functions occurs when $A(x)$ is decreasing.

Now we begin determining what all this means and reducing the problem further. Consider the difference equation

$$
B\left(x_{t}\right)=A\left(x_{t+1}\right)
$$

from above and recall that we are interested in the solutions to the difference equation, which are sequences $x_{0}, x_{1}, x_{2}, \ldots$ of nonnegative real numbers satisfying the difference equation. Both $A$ and $B$ are continuous functions from $[0, \infty)$ to $[0, \infty)$. The functions $A$ and $B$ have the following properties:

1. While $B$ is increasing and therefore one-to-one, $A$ is not one-to-one.

2. For some positive number $c$, both $A$ and $B$ are linear on $[c, \infty)$ with positive slopes, and the slope of $\left.A\right|_{[c, \infty)}$ is less than the slope of $\left.B\right|_{[c, \infty)}$.

3. On some interval $[0, b]$, (with $b<c$ ) the behavior of $A$ may be increasing with $A(0)=0$ (case I), or it may be increasing with $A(0)>0$ (case II), or $A$ may be decreasing on $[0, c]$ (case III). For case III we let $b=0$.

4. On the interval $(b, c], A$ is decreasing, with $x \in(b, c)$ such that $A(x)=B(x)$.

Note that there are positive numbers $\underline{x}$ and $\bar{x}$ such that

$$
\begin{aligned}
& B(\underline{x})=A(c), \\
& B(\bar{x})=A(\underline{x})
\end{aligned}
$$

and in case I and perhaps case II, there are positive numbers $\underline{x}^{b}$ and $\bar{x}^{b}$ such that

$$
\begin{aligned}
& B\left(\bar{x}^{b}\right)=A(b), \\
& B\left(\underline{x}^{b}\right)=A\left(\bar{x}^{b}\right) .
\end{aligned}
$$

Since the function $A$ is not one-to-one, the dynamics in the model given by the difference equation (10) are not well-defined. However, since $B$ is one-to-one, we can invert $B$ and define the function $f(x):=$ $B^{-1} \circ A(x)$. This function gives the backward dynamics $x_{t}=f\left(x_{t+1}\right)$, maps $[0, \infty)$ to itself and inherits the basic shape of $A$. Consequently, even though the dynamics of (10) are not well-defined going forward in time, the dynamics are well-defined going backward in time. In terms of the $f$ function we have:

$$
\begin{aligned}
\underline{x} & :=f(c), \\
\bar{x} & :=f(\underline{x}), \\
\bar{x}^{b} & :=f(b), \\
\underline{x}^{b} & :=f\left(\bar{x}^{b}\right) .
\end{aligned}
$$



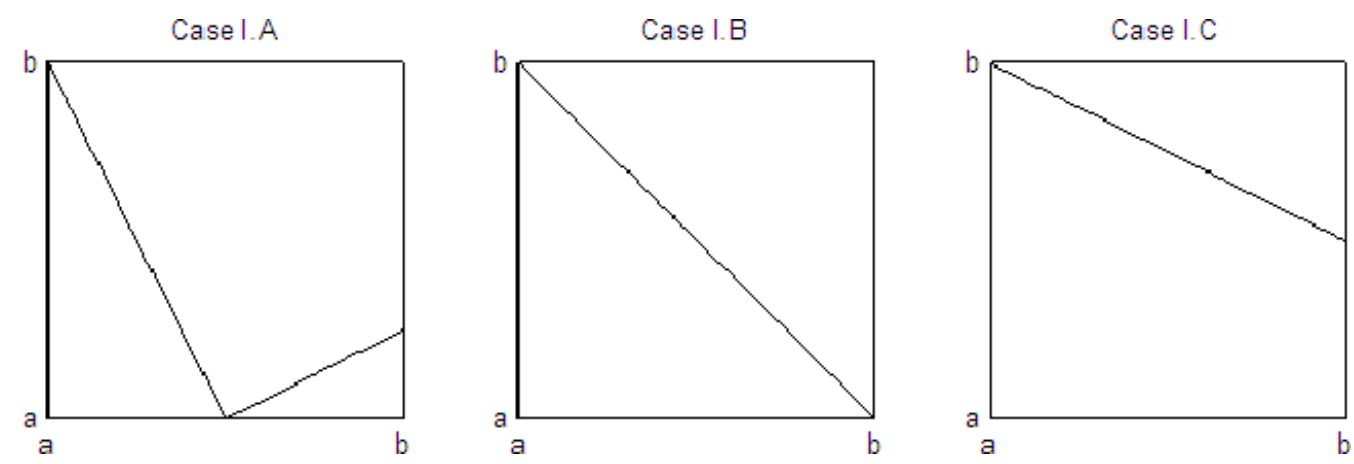

Figure 4. Case I

Remark 1 If $b>0$, we have $A(\underline{x}) \leq A(b)$, which implies

$$
B(\bar{x})=A(\underline{x}) \leq A(b)=B\left(\bar{x}^{b}\right) .
$$

Since $B$ is increasing, this implies $\bar{x} \leq \bar{x}^{b}$.

Remark 2 If $b>0$, we have $A\left(\bar{x}^{b}\right) \geq A(c)$ (since $\left.\bar{x}^{b}>b\right)$, which implies

$$
B(\underline{x})=A(c) \leq A\left(\bar{x}^{b}\right)=B\left(\underline{x}^{b}\right) .
$$

Since B is increasing, this implies $\underline{x} \leq \underline{x}^{b}$

In [66], it is shown that there are three generic possibilities for $f$ in Case I where $0<b \leq \underline{x}$ :
I.A. $\bar{x}>c$
I.B. $\bar{x}=c$
I.C. $\bar{x}<c$

See Figure 4 for an illustration of the three possibilities for Case I.

Michener and Ravikumar also show (see [66]) that there are three generic possibilities for $f$ in Case II where $\underline{x}<b$ :

II.A. $\underline{x} \leq \underline{x}^{b}<b<\bar{x}^{b} \leq c$.

II.B. $\underline{x}<b \leq \underline{x}^{b}<\bar{x}^{b} \leq c$.

II.C. $\underline{x}<b<c<\underline{x}^{b}$.

See Figure 5 for an illustration of these three possibilities for Case II.

The dynamics are not interesting in cases I.B, I.C, and II.B. The three-cycle proposition in [66 p. 1128] assumes $(1+\theta) c / \beta \leq \bar{x}$. Since $(1+\theta)>\beta$ (by assumption in [66]), these sufficient conditions for chaos only cover cases I.A and II.C. [66] illustrate by an example that chaos is possible in case II.A.

Thus far, our work has dealt with case I.A only. Cases I.B and II.B are the same and not interesting, and case I.C is not interesting either. Case II.A is interesting and case II.C (sort of a combination of Cases I.A and II.A) is interesting also. Cases II.A and II.C should be topics for future study.

Thus, we know quite a bit about the possible behavior of $A, B$, and $f$. However, there is still much not known: different choices of $A$ and $B$ all satisfying the required conditions yield very different dynamical behavior. Some choices lead to rather boring dynamical systems, and some lead to interesting ones. 

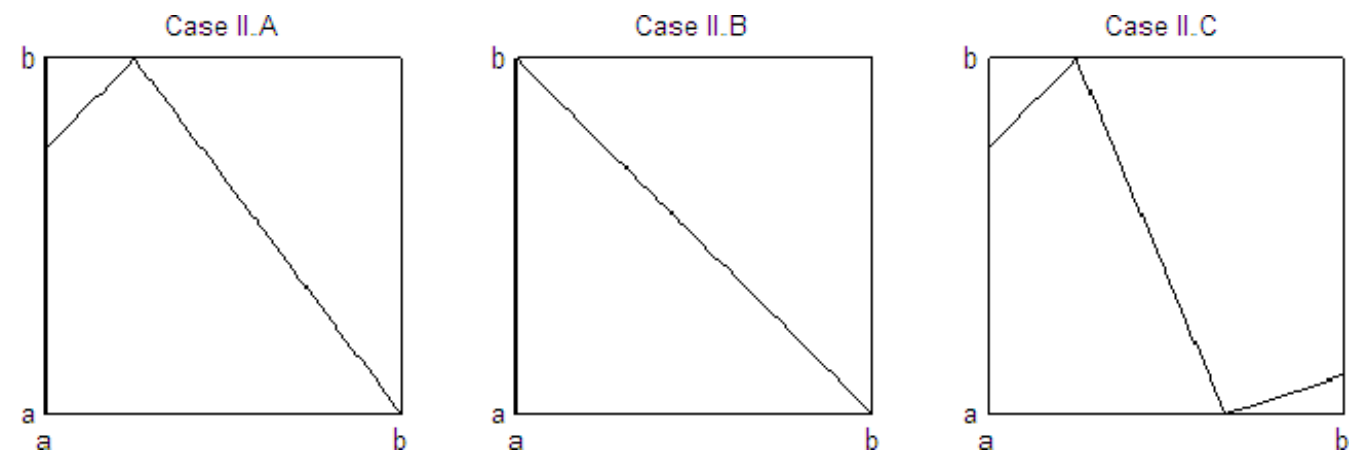

Figure 5. Case II

One obvious equilibrium is the sequence $\left(x^{*}, x^{*}, x^{*}, \ldots\right)$, where $x^{*}$ is the positive number such that $A\left(x^{*}\right)=B\left(x^{*}\right)$. We will call this the trivial solution. There are many other solutions. For case I.A, note that if a sufficiently large $x_{0}$ is chosen, then the requirement that $A\left(x_{1}\right)=B\left(x_{0}\right)$ forces $x_{1}$ to be larger than $x_{0}$, and $x_{1}$ is unique. (See Figure 2]) Continuing, one sees that the solution $\left(x_{0}, x_{1}, \ldots\right)$ for that initial condition is well-defined and $\lim _{t \rightarrow \infty} x_{t}=\infty$. Likewise, if a sufficiently small positive $x_{0}$ is chosen, then $x_{1}$ is smaller than $x_{0}$, and the solution $\left(x_{0}, x_{1}, \ldots\right)$ consists of a decreasing sequence of positive numbers converging to 0 . We summarize the possibilities precisely in the following propositions.

As before, let $\underline{x}$ denote the unique positive number such that $B(\underline{x})=A(c)$. Let $\bar{x}$ denote the unique positive number such that $B(\bar{x})=A(\underline{x})$. Then for the cases we consider, $\underline{x}<c<\bar{x}$.

Proposition 1 Consider case I.A. If $\left(x_{0}, x_{1}, \ldots\right)$ is a solution to $A\left(x_{t+1}\right)=B\left(x_{t}\right)$ such that $x_{\widehat{t}}<\underline{x}$ for some $\widehat{t}$, then

[a] for $t \geq \widehat{t}$, the choice of $x_{t+1}$ is unique, i.e., $x_{t+1}$ such that $A\left(x_{t+1}\right)=B\left(x_{t}\right)$ is unique;

[b] $\lim _{t \rightarrow \infty} x_{t}=0$; and

[c] $x_{\widehat{t}}>x_{\widehat{t}+1}>x_{\widehat{t}+2}>\cdots$.

Proposition 2 For case I.A, if $\left(x_{0}, x_{1}, \ldots\right)$ is a solution to $A\left(x_{t+1}\right)=B\left(x_{t}\right)$ such that $x_{\widehat{t}}>\bar{x}$ for some $\widehat{t}$, then the choice of $x_{\widehat{t}+1}$ may not be unique, but either

[a] $\lim _{t \rightarrow \infty} x_{t}=\infty$ and eventually $x_{t}<x_{t+1}<x_{t+2}<\cdots$, or

[b] $\lim _{t \rightarrow \infty} x_{t}=0$ and eventually $x_{t}>x_{t+1}>x_{t+2}>\cdots$.

It follows from the previous propositions that in case I.A solutions that contain members not in the interval $[\underline{x}, \bar{x}]$ exhibit simple behavior. Mathematically they are not very interesting. From an economics perspective, they may not constitute an equilibrium (the transversality condition may be violated). If the transversality condition is satisfied in these cases, then such equilibria are referred to as self-fulfilling inflations $\left(x_{t} \rightarrow 0\right)$ and self-fulfilling deflations $\left(x_{t} \rightarrow \infty\right)$. Moreover, a solution containing a member not in $[\underline{x}, \bar{x}]$ would be locked into one behavior-either its members would eventually increase without bound, or they would eventually decrease to 0 . We can also conclude that solutions to (10) that satisfy $0<\underline{x}<x_{t}<\bar{x}<\infty$ for all $t$ will be an equilibrium in the model.

Note that the original model with its equilibria defined as a collection $\left\{c_{1 t}, c_{2 t}, m_{t}, M_{t}, p_{t}\right\}_{t=0}^{\infty}$ of sequences that satisfy conditions (2)-(7) and Assumptions 1-3 has now been reduced to an implicitly defined difference equation on an interval, and then to the function $f$ from the positive reals to the positive reals. Although $f$ has the "problem" of backward dynamics, the problem of understanding the model has certainly been reduced. An equilibrium in the reduced model is now just a sequence of nonnegative numbers $\left\{x_{t}\right\}_{t=0}^{\infty}$ 
such that $f\left(x_{t+1}\right)=x_{t}$. Furthermore, since any equilibrium that contains a member not in $[\underline{x}, \bar{x}]$ behaves in a simple and completely predictable manner dynamically, and is not good news economically (if it even satisfies the transversality condition), we may as well restrict ourselves to the study of only those sequences each member of which is in the interval $[\underline{x}, \bar{x}]$. We call the restricted version of $f, f$, also. Thus, we are down to studying interval dynamics (or are we?), although they are backward interval dynamics.

\section{The Overlapping Generations model}

The overlapping generations model (OLG model) is a type of economics model in which agents live a finite amount of time and live long enough to endure into at least one period of the next generations' lives. The concept of OLG was developed in 1947 by Maurice Allais and popularized by Paul Samuelson in 1958. OLG models can have varying characteristics depending on the method of study but many models share several key elements. (We thank Wikipedia for this nice list.)

- Individuals receive an endowment of goods at birth.

- Goods cannot endure for more than one time period.

- Money endures for more than one period.

- Individuals must consume in all periods and their lifetime utility is a function of consumption in all periods.

- Individuals live for two periods-in the first period they are called the Young; in the second they are the Old.

- A number of individuals is born in every period with the specific number born in a given period $t$ denoted as $N_{t}$. (Thus, $N_{1}$ denotes the people born in period 1.)

- The economy begins in period 1 , and in period 1 there is a group of people who are already old. They are the initial Old and are denoted as $N_{0}$.

- There is only one good and it cannot endure for more than one period.

- Each individual receives a fixed endowment of the good at birth. This endowment is denoted as $y$. It can also be thought of as an endowment of labor that the individual uses to work and create a real income equal to the value of good $y$ produced. Under this framework, individuals only work during the young phase of their lives.

Next we sketch the OLG model derivation for the particular OLG model studied by Raines and Medio. For more details, see [63].

In each generation, the young representative agent maximizes utility of consumption over the twoperiod life, subject to the constraint that the total value of consumption must be no greater than the total value of the endowments received. Also, the market clearing condition holds, that is, the market for the consumption good is always in equilibrium, which means here that in each period the demand for the consumption good from young and old is equal to the total supply, namely the total endowments, and that perfect foresight holds, that is, agents' expectations are always fulfilled. Another assumption is that the population is constant: for each period the number of Young is, say, $N$, which is also the number of Old, and therefore the total population number is $2 N$.

Let $c_{t} \geq 0$ be the young agent's consumption at time $t$, and let $d_{t} \geq 0$ be the old agent's consumption at time $t$. Let $e_{y n g} \geq 0$ be the young agent's endowment and $e_{\text {old }} \geq 0$ be the old agent's endowment. The basic utility functions are $u_{1}$ and $u_{2}$, with $u_{1}$ a function of the young agent's consumption $c_{t}$ and $u_{2}$ a function of the old agent's consumption $d_{t}$. Now define another utility function $U$ by $U\left(c_{t}, d_{t}\right)=u_{1}\left(c_{t}\right)+u_{2}\left(d_{t+1}\right)$. (Thus, $U$ is formed by adding the respective basic utilities of the young and old agents.) 
Let $p_{t} \geq 0$ be the interest factor at time $t$, that is, the exchange rate between present and future consumption. Note that $d_{t}$ and $c_{t}$ must be nonnegative, because negative consumption makes no sense. A mathematical formulation of the problem then is to maximize the function $u_{1}\left(c_{t}\right)+u_{2}\left(d_{t+1}\right)$ such that $d_{t+1} \leq e_{\mathrm{old}}+p_{t}\left(e_{\mathrm{yng}}-c_{t}\right)$ and $c_{t}, d_{t+1} \geq 0$. The market clearing condition for all time $t$ is $c_{t}+d_{t}=e_{\mathrm{yng}}+e_{\mathrm{old}}$, that is, total consumption is equal to total income (endowment).

Economists are interested in studying the properties of infinite sequences of $c_{t}$ (or equivalently, of $d_{t}$, satisfying the optimality conditions and market clearing condition. From the first order conditions of the constrained maximization and the market clearing condition, it follows (some details are left out heresee [63]) that the young agent's optimal choice must satisfy the equation $H\left(d_{t+1}, c_{t}\right)=\mathcal{U}\left(d_{t+1}\right)+\mathcal{V}\left(c_{t}\right)=$ 0 where $\mathcal{U}(d)=u_{2}^{\prime}(d)\left(e_{\text {old }}-d\right)$ and $\mathcal{V}(c)=u_{1}^{\prime}(c)\left(e_{\mathrm{yng}}-c\right)$.

Whether or not we can now derive a difference equation moving forward in time depends on whether the function $\mathcal{U}$ is invertible. Consider the following specific example:

$$
u_{1}(c)=a c-(b / 2) c^{2} ; u_{2}(d)=d
$$

where $a$ and $b$ are positive constants. In this case, $\mathcal{U}(d)=e_{\mathrm{old}}-d$ is of course invertible. For simplicity's sake and without loss of generality, we put $e_{\mathrm{yng}}=0$ and $a=b=\mu$. Then $e_{\mathrm{old}}-d_{t+1}=c_{t+1}=F_{\mu}\left(c_{t}\right)=$ $\mu c_{t}\left(1-c_{t}\right)$, a much studied noninvertible map. Starting from an arbitrary initial condition $c_{0} \in[0,1]$, this equation determines sequences of young agents' consumption forward in time. Applying the equilibrium condition $c_{t}+d_{t}=e_{\mathrm{yng}}+e_{\mathrm{old}}$, the old agents' consumption is determined as well.

The case on which Medio and Raines focus: Suppose that we now interchange the utility functions, so that $u_{1}(c)=c$ and $u_{2}(d)=a d-(b / 2) d^{2}$ and then put $e_{\mathrm{old}}=0, e_{\mathrm{yng}}>0$. In this case, we can instead write an equation

$$
e_{\text {yng }}-c_{t}=d_{t}=F_{\mu}\left(d_{t+1}\right)=\mu d_{t+1}\left(1-d_{t+1}\right)
$$

so that $F_{\mu}$ now defines sequences of old agents' consumption (and by implication, young agents' consumption) moving backward in time.

But now we have a problem. Economically, it means that to each value of the young agent's present saving (endowment minus consumption) there may correspond two or more values of expected future consumption that justify it—or, if $\mu<4$, there may be none. (See Figure 6) This problem will occur with great generality whenever the function $\mathcal{U}$ is noninvertible. Nor is it limited to this version of OLG but it may also occur in two-dimensional models of OLG with production, i.e., models in which consumption is produced by means of current labor and capital stock invested one period ago. In order to have a visual insight of the nature of the problem when $e_{\text {yng }}-c_{t}=d_{t}=F_{\mu}\left(d_{t+1}\right)$ holds, consider the figure (logistic map), where we represent the curve of the function $F_{\mu}$ in the plane $\left(d_{t+1}, d_{t}\right)$ and for simplicity's sake assume that $d_{\max }=e_{\text {yng }}$.

Start at time $t=0$, and suppose that our maximizing young agent considers the possibility of consuming an amount $c_{0}$ and thereby saving an amount $e_{\mathrm{yng}}-c_{0}=d_{0}$. The preimage of $d_{0} \in\left[0, d_{\max }=e_{\mathrm{yng}}\right]$, $F_{\mu}^{-1}\left(d_{0}\right)$, consists of two points, the lesser of which we label $d_{1}^{0}$ and call low-level consumption and the higher of which we label $d_{1}^{1}$ and call high-level consumption. Hence there are two levels of future consumption at time 1 that would be allowed for the young agent at time 0 . This problem continues: At time 2 , there are two levels of future consumption allowed for the young agent at time 1 , and so on. If $d_{0} \in\left(e_{\mathrm{yng}}, 1\right]$, then there is no preimage of $d_{0}$, that is, $F_{\mu}^{-1}\left(d_{0}\right)$ is empty.

Hence, not all sequences of consumption are admissible, that is, some choices lead to a sequence that must at some point stop, because the preimage of the last member of the previously chosen finite sequence is empty. Thus, we make the following definition: We say an infinite sequence $\left\{d_{t}\right\}_{t=0}^{\infty}$ is forward admissible if $d_{t} \geq 0$ and $d_{t}=F_{\mu}\left(d_{t+1}\right)$ for all $t \geq 0$.

\section{Tools from mathematics}

Both the CIA and OLG models studied have now been reduced to the study of a unimodal map $h$ on an interval $J$ of the form $h\left(x_{t+1}\right)=x_{t}$ for $x_{t} \in J$. Both are thus well-defined backward in time, but not 


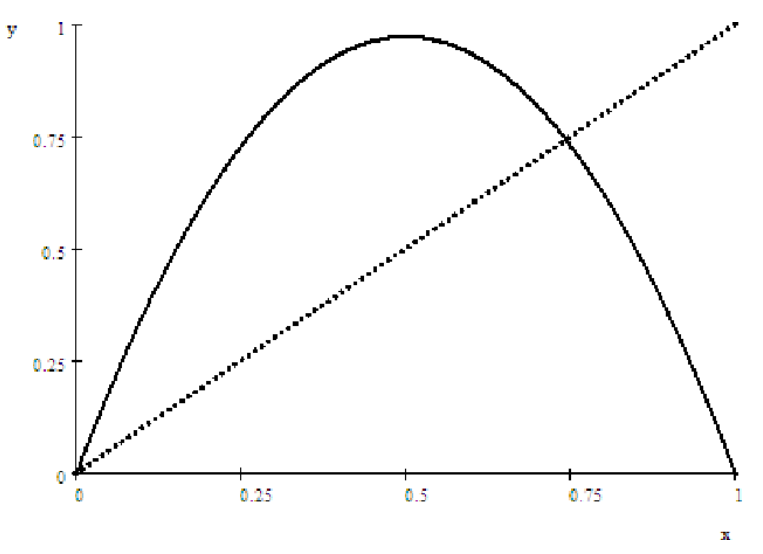

Figure 6. Backward moving map $F_{\mu}$ with $\mu<4$.

forward in time. In both cases, economists are not interested in one number $x_{t}$, but in entire sequences of $\left(x_{0}, x_{1}, \ldots\right)$ such that $h\left(x_{t+1}\right)=x_{t}$ for $t$ a nonnegative integer, since these sequences form the equilibria for both models. It occurred to all of us that the map $h$ on the interval $J$ was only part of the story: our equilibria sit in the inverse limit space $\lim (J, h)$. This is the space we should be studying: the dynamics on it are intimately related to the dynamics of $h$ on $J$ since $h: J \rightarrow J$ generates $\lim (J, h)$. Furthermore, we have an induced homeomorphism $h^{*}$ on $\lim (J, h)$. And-a real bonus, topologists and dynamicists have been studying these limit spaces for over 50 years, and there is a vast literature we can just "plug into". (See

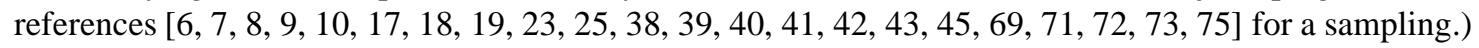

Suppose $\mathbb{N}$ denotes the positive integers and $\widetilde{\mathbb{N}}$ denotes the nonnegative integers. A continuum is a compact, connected metric space. If $X$ and $Y$ are continua, and $Y \subset X$, then $Y$ is a subcontinuum of $X$. If $Y$ is a subcontinuum of $X$, but $Y \neq X$, then $Y$ is a proper subcontinuum of $X$.

A chain is a finite sequence $G_{1}, G_{2}, \ldots, G_{n}$ of open sets such that $G_{i}$ intersects $G_{j}$ if and only if $|i-j| \leq 1$. The open sets $G_{i}$ are the links of the chain. The mesh of the chain is the largest diameter of its links. A continuum is chainable provided for each positive integer $\epsilon$ there is a chain cover of $M$ with mesh less than $\epsilon$. The unit interval is the most obvious example of a chainable continuum, but there are examples that are much more interesting.

A continuum is decomposable if it is the union of two of its proper subcontinua. If a continuum is not decomposable, it is indecomposable. A reader not familiar with these objects might wonder whether they exist. They do indeed, and are quite common occurrences in chaotic dynamical systems. All indecomposable continua share certain structure. If $X$ is an indecomposable continuum and $x \in X$, then $\operatorname{Cps}(x)=\{y \in X$ : there is a proper subcontinuum of $X$ that contains both $x$ and $y\}$; $\operatorname{Cps}(x)$ is called the composant of $x$. The set of composants of an indecomposable continuum partitions the continuum into an uncountable collection of mutually disjoint sets, each of which is dense in the continuum. Each composant is like a "highway" in the continuum. The continuum is made from the collection of highways, each close to any other but forever apart from the other.

A continuum with the property that every proper subcontinuum is an arc is called an arc continuum. Note that a continuum can be both indecomposable and an arc continuum. A continuum can also be both indecomposable and chainable. In the case of an indecomposable arc continuum, each composant of the indecomposable arc continuum is an arc component. (If $X$ is an $\operatorname{arc}$ continuum, and $x \in X$, the $\operatorname{arc}$ component $A(x)$ of the point $x$ is the set $\left\{z \in X\right.$ : there is an $\operatorname{arc} P_{z}$ in $X$ that contains both $x$ and $\left.z\right\}$.) The familiar Knaster bucket handle continuum, which is homeomorphic to the Smale horseshoe attractor, is a chainable, indecomposable arc continuum. However, indecomposable arc continua need not be chainablethe solenoids are indecomposable arc continua, but they are not chainable. Likewise, indecomposable chainable continua need not be arc continua. The pseudoarc is an indecomposable chainable continuum 
which contains no arcs. In fact, every proper subcontinuum of a pseudoarc is either a point or a copy of the pseudoarc.

Suppose $X$ is a compact metric space and $f: X \rightarrow X$ is continuous. The point $x$ in $X$ is a periodic point of period $n$ if $f^{n}(x)=x$. If $x$ is a periodic point of period $n$, then its orbit is $O_{+}(x):=$ $\left\{x, f(x), f^{2}(x), \ldots, f^{n-1}(x)\right\}$. We can talk about the orbits of nonperiodic points, too: If $X$ is a metric space and $f: X \rightarrow X$ is continuous, the orbit $O_{+}(x)$ of the point $x$ under the action of $f$ is the set $O_{+}(x)=\left\{x, f(x), f^{2}(x), \ldots\right\}$.

A subset $A$ of $X$ is invariant under $f$ if $f(A)=A$. (Hence the orbit of a periodic point is an invariant subset of $X$ under $f$.)

Suppose that $X$ and $Y$ are metric spaces, $f: X \rightarrow X$ is continuous and $g: Y \rightarrow Y$ is continuous. If there is a homeomorphism $h: X \rightarrow Y$ such that $h \circ f=g \circ h$, then $f$ and $g$ are said to be conjugate. Whenever two maps are conjugate, their dynamics are equivalent. A weaker condition is that of semiconjugacy: If there is a continuous onto map $h: X \rightarrow Y$ such that $h \circ f=g \circ h$, then $f$ and $g$ are said to be semi-conjugate, and $f$ factors over $g$. If two maps are semi-conjugate, their dynamics are related, but not necessarily equivalent.

A subset $A$ of a complete, separable metric space $X$ is residual in $X$ if $A$ contains a dense $G_{\delta}$ subset of $X$. A subset $M$ of $X$ is nowhere dense in $X$ provided the interior of the closure of $M$ in $X$ is empty. $M$ is meager in $X$ if $M$ is a countable union of nowhere dense subsets of $X$. The complement of a meager set in a complete separable metric space is a residual set of that space.

Suppose that $K$ is a compact, metric space, and $h: K \rightarrow K$ is continuous. If there is a point $p$ which has a dense orbit in $K$ under the action of $h$, then there is a residual set of points in $K$ each of which has its orbit dense in $K$. We say that $h$ is transitive if there is a point $p$ in $K$ which has its orbit dense in $K$. The map $h$ is transitive if and only if it has the following property: if $u$ and $v$ are nonempty open subsets of $K$, then there is some integer $n$ such that $h^{n}(u) \cap v \neq \varnothing$. The map $h$ has sensitive dependence on initial conditions on the invariant closed subset $H$ of $K$ if there is some positive number $r$ such that for each point $x$ in $H$ and for each $\epsilon>0$, there is a point $y$ in $H$ with $d(x, y)<\epsilon$ and an integer $k \geq 0$ such that $d\left(h^{k}(x), h^{k}(y)\right) \geq r$. The map $h$ is chaotic in the sense of Li and Yorke if $h$ has sensitive dependence on initial conditions on $K$. The map $h$ is chaotic in the sense of Devaney if

1. there is a point $p$ in $K$ which has its orbit dense in $K$,

2. the set of periodic points in $K$ is dense in $K$, and

3. $h$ is sensitive to initial conditions at each point of $K$.

If $X$ is a metric space and $f: X \rightarrow X$ is continuous, $f$ is transitive, and the set of periodic points of $f$ is dense in $X$, then $f$ has sensitive dependence on initial conditions [5]. Thus, Devaney's last condition is redundant. Rob Roe [75] has shown that if $X$ is a finite tree and $f: X \rightarrow X$ is continuous and has a dense orbit, then $f$ is chaotic in the sense of Devaney. (Note that Roe's assumption that $X$ be a tree is important: An irrational rotation on a circle forms a dynamical system in which every orbit is dense, but it is not chaotic and it does not have any periodic points.) Thus, for a map from an interval to itself, condition 1 above implies that the map is chaotic in the sense of Devaney.

Suppose that $x$ is a fixed point of $f$ (i.e., $f(x)=x$ ). We say that $y$ is homoclinic to the fixed point $x$ if there is $y \neq x$ such that $f^{n}(y) \rightarrow x$ and there is a choice of inverse images $f^{-1}(y), f^{-2}(y), \ldots$ with $f^{-n}(y) \rightarrow x$. If $x$ is a periodic point of period $n$ under $f$, we say that $y$ is homoclinic to $x$ if $y$ is homoclinic to $x$ under $f^{n}$.

Let $f: I \rightarrow I$ be a continuous map on an interval $I$ such that $I=[a, b]$. We say that $f$ has a (onedimensional) horseshoe if there are disjoint subintervals $I_{0}$ and $I_{1}$ of $I$ and $n_{0} \in \mathbb{N}$ such that $I_{0} \cup I_{1} \subset$ $f^{n}\left(I_{0}\right)$ and $I_{0} \cup I_{1} \subset f^{n}\left(I_{1}\right)$.

The notion of topological entropy involves the concept of an $(n, \epsilon)$-separated set. Let $f$ be a continuous map on a compact metric space $X$ with metric $d$. Let $A, E \subset X$. We say that $E$ is $(d, \epsilon, A)$-spanning if $E$ is finite and for every $y$ in $A$, there exists an $x \in E$ such that $d(x, y)<\epsilon$. Given $f$, for $n \in \widetilde{\mathbb{N}}$, 
we define a new metric $d_{n}^{f}$ on $X$ given by $d_{n}^{f}(x, y):=\max _{0 \leq i \leq n-1} d\left(f^{i}(x), f^{i}(y)\right)$. For $n \in \widetilde{\mathbb{N}}$ and $\epsilon>0$, let $S\left(d_{n}^{f}, \epsilon, A\right)$ denote the minimum cardinality of all $\left(d_{n}^{f}, \epsilon, A\right)$-spanning sets. Then define for $A \subset X, h(f, A, \epsilon)=\limsup _{n \rightarrow \infty}(1 / n)\left[\log S\left(d_{n}^{f}, \epsilon, A\right)\right]$. The topological entropy of $f$ on $A$ is defined by $h(f, A)=\lim _{\epsilon \rightarrow 0} h(f, A, \epsilon)$. The topological entropy of $f$ is defined by $h(f)=h(f, X)$.

A continuum $X$ has the fixed point property if for every continuous function $h$ from $X$ to itself, there is a point $p$ in $X$ such that $h(p)=p$.

A map of an interval onto itself is called Markov if there is a finite invariant set $A$ containing the end points of the interval such that if $p$ and $q$ are consecutive members of $A$, then the restriction of the map to $[p, q]$ is monotone.

A topological ray is a locally compact, connected metric space $R$ containing a point $O$ such that $R \backslash\{O\}$ is connected, and if $p \in R$, but $p \neq O$, then $R \backslash\{p\}$ is the union of two disjoint connected sets.

Unfortunately, there is no commonly accepted definition of the word "attractor", and to make matters worse, Kennedy-Stockman-Yorke use the word one way and Medio-Raines use it another way. Let $X$ be a complete separable metric space and $f: X \rightarrow X$ be continuous. Let $A \subseteq X$ such that $A$ is a closed invariant set. We say $A$ is an attractor for $X$ if there is an open set $O$ such that $A \subseteq O$ and $\cap_{n=0}^{\infty} f^{n}(O)=A$. This is how Kennedy-Stockman-Yorke use the word.

Now for the Medio-Raines version: Again, let $f: X \rightarrow X$ be a continuous map on the metric space $X$. The $\omega$-limit set of a point $x$ of $X$ is defined to be $\omega_{f}(x)=\cap_{i \geq 0} \overline{\left\{f^{m}(x): m \geq i\right\}}$. If $A$ is closed and invariant under $f$, then the basin of attraction of $A$ is defined to be $\mathcal{B}(A)=\left\{x \in X: \omega_{f}(x) \subseteq A\right\}$, and $A$ is called a topological attractor provided $\mathcal{B}(A)$ contains a residual subset of an open subset of $X$ and there is no closed invariant subset $A^{\prime}$ of $A$ for which $\mathcal{B}(A)$ and $\mathcal{B}\left(A^{\prime}\right)$ coincide up to a meager set. However, $A$ is a metric attractor provided $\mathcal{B}(A)$ has positive measure and there is no closed invariant subset $A^{\prime}$ of $A$ for which $\mathcal{B}(A)$ and $\mathcal{B}\left(A^{\prime}\right)$ coincide up to a set of measure zero. An attractor $A$ is Liapunov stable provided there are arbitrarily small neighborhoods $O$ of $A$ such that $f(O) \subseteq O$. If $A$ is Liapunov stable and its basin of attraction is open and nonempty, then $A$ is asymptotically stable.

A map of a continuum to itself is monotone provided each point inverse is a continuum. A piecewise monotone function on an interval $[a, b]$ is expanding if whenever $x<y$ and $f$ is monotone on $[x, y]$, then

$$
\frac{|f(y)-f(x)|}{|y-x|}>1 .
$$

If $[a, b]=\left[r_{0}, r_{1}\right] \cup\left[r_{1}, r_{2}\right] \cup \cdots \cup\left[r_{n-1}, r_{n}\right]$ and $f$ is strictly increasing or strictly decreasing on each subinterval $\left[r_{i-1}, r_{i}\right]$, let $\mathcal{T}=\left\{J_{0}, J_{1}, \ldots, J_{2 n}\right\}$, where $J_{2 i}=\left\{r_{i}\right\}$ for $0 \leq i \leq n$, and $J_{2 i+1}=\left(r_{i}, r_{i+1}\right)$ for $0 \leq i \leq n-1$. We say $x$ and $y$ have different itineraries if $f^{n}(x)$ and $f^{n}(y)$ are in different members of $\mathcal{T}$ for some $m \geq 0$. A piecewise monotone map on an interval $[a, b]$ is weakly expanding if, whenever $x \neq y$ in $[a, b], x$ and $y$ have different itineraries.

A map $f$ of an interval $[a, b]$ onto an interval $[d, e]$ is unimodal provided $f$ is not monotone, and there is a point $c$ in $(a, b)$ such that $\left.f\right|_{[a, c]}$ and $\left.f\right|_{[c, b]}$ are both monotone. The point $c$ is called the turning point for $f$. The map $f$ is a Type (1) unimodal map if $f(b)=a$. Several families of tent maps (piecewise linear unimodal maps) and logistic maps (of the form $F_{\mu}(x)=\mu x(1-x)$-such as those associated with the OLG model) have been extensively studied by a number of authors. (See [8, 6, 39, 43], for example.)

Type (1) unimodal maps go up and then come down; members of our CIA family of maps go down and then come up. This is not a problem: we can "flip" our map over so as to more easily use the results in the literature. Also, translating to $[0,1]$ from the interval $[\underline{x}, \bar{x}] \subset(0, \infty)$ (via a conjugacy) is easy. Thus, our CIA interval maps are all conjugate to type (1) unimodal maps.

In the OLG work, the following definitions are used: Let $f$ be continuous with domain $[0,1]$. Then we say that $f$ is

(1) a type $A$ unimodal map provided $f$ is unimodal on $[0,1]$ with turning point $c, f(0)=0$ and $f(c) \leq 1$,

(2) a type $B$ unimodal map provided $f$ is unimodal on $[0,1]$ with turning point $c, f(0)>0$ and $f(c) \leq 1$, 
(3) a type $C$ unimodal map provided there is a point $c \in(0,1)$ such that $\left.f\right|_{[0, c]}$ is strictly increasing, $\left.f\right|_{[c, 1]}$ is strictly decreasing, and $f(c)>1$.

Suppose $f:[a, b] \rightarrow[a, b]$ is continuous and onto. We say $f$ has finitely many turning points if there exists a finite set $\left\{a_{0}, a_{1}, \ldots, a_{n}\right\}, a=a_{0}<a_{1}<\cdots<a_{n}=b$ such that $f$ is monotone on $\left[a_{i-1}, a_{i}\right]$ for $i=1, \ldots, n$, but $f$ is not monotone on any interval in $[a, b]$ properly containing some $\left[a_{i-1}, a_{i}\right]$. Members of the set $\left\{a_{1}, \ldots, a_{n-1}\right\}$ are the turning points for $f$.

Let, for each $m \in \widetilde{\mathbb{N}}, X_{m}$ be a nonempty metric space, and $f_{m}$ denote a continuous map from $X_{m+1}$ to $X_{m}$. The pair $\left(X_{m}, f_{m}\right)$ is called an inverse sequence or inverse system. The spaces $X_{m}$ are called factor spaces, and the mappings $f_{m}$ are called bonding maps.

Suppose $\left(X_{m}, f_{m}\right)$ is an inverse sequence. The inverse limit of the inverse sequence is denoted by $\lim _{L}\left(X_{m}, f_{m}\right)$ and is defined as the subset of the product space $\prod_{m \in \mathbb{N}} X_{m}$, to which the point $\mathbf{x}$ (with

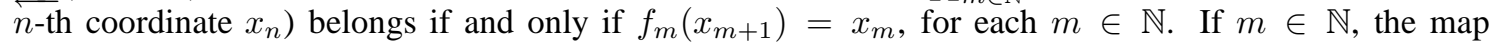
$\pi_{m}: \prod_{n \in \mathbb{N}} X_{n} \rightarrow X_{m}$ defined by $\pi_{m}(\mathbf{x})=x_{m}$ is called the projection map (or, if specificity is required the $m$-th projection map).

We note that inverse systems and inverse limits can be defined for a much broader class of spaces (here we limit ourselves to metric spaces) and indexing sets (here we use the nonnegative integers for indexing), but the definitions above are sufficient for our purposes. In fact, except when we are talking about subsets of inverse limits, our inverse limit spaces here are all generated with the space a closed interval J, and the map a continuous map $f: J \rightarrow J$ (so just one bonding map), so our inverse limit spaces are of the form $\underset{\lim }{\longleftarrow}(J, f)$. Some useful background theorems we need about the properties of inverse limit spaces are given

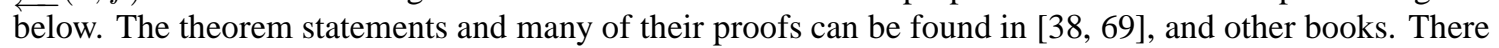
are many more such theorems.

Theorem 1 If $\left(X_{m}, f_{m}\right)$ is an inverse sequence and each $X_{m}$ is compact, then its inverse limit is a nonempty compact metric space contained in $\prod_{m \in \mathbb{N}} X_{m}$. If $\left(X_{m}, f_{m}\right)$ is an inverse sequence and for each $m \in \mathbb{N}, X_{m}$ is a continuum, then the inverse limit of the inverse sequence is a continuum. If $\left(X_{m}, f_{m}\right)$ is an inverse sequence and each $X_{m}$ is a chainable continuum, then $\lim \left(X_{m}, f_{m}\right)$ is a chainable continuum, is of topological dimension 1, can be embedded in the plane, and has the fixed point property.

Theorem 2 If $\left(X_{m}, f_{m}\right)$ is an inverse sequence and $X=\lim _{\longleftarrow}\left(X_{m}, f_{m}\right)$, then $\left.\pi_{m}\right|_{X}: X \rightarrow X_{m}$ is continuous.

Theorem 3 If $\left(X_{m}, f_{m}\right)$ is an inverse sequence, and $X$ is the inverse limit of the inverse sequence, then for each $m<n \in \mathbb{N},\left.\pi_{m}\right|_{X}=f_{m} \circ f_{m+1} \circ \cdots \circ f_{n-1} \circ f_{n} \circ\left(\left.\pi_{n}\right|_{X}\right)$. (Often the composition map $f_{m} \circ f_{m+1} \circ \cdots \circ f_{n-1} \circ f_{n}$ is denoted $f_{m}^{n}$ and $f_{m}^{m}$ is used to denote the identity on $X_{m}$. The notation makes bookkeeping easier.)

Theorem 4 If $\alpha_{1}, \alpha_{2}, \ldots$ is a sequence of arcs each of which is a proper subset of a continuum $X$ such that $\alpha_{1} \subset \alpha_{2} \subset \alpha_{3} \subset \cdots$, the point $O$ is a common endpoint of $\alpha_{1}, \alpha_{2}, \ldots, R=\alpha_{1} \cup \alpha_{2} \cup \alpha_{3} \cup \cdots$, and no point of $\alpha_{n}$ belongs to $\overline{R \backslash \alpha_{n+1}}$, then $R$ is a ray.

Theorem 5 If $\left(X_{m}, f_{m}\right)$ is an inverse limit system such that for each $m, X_{m}$ is a compact metric space and $f_{m}$ is a homeomorphism, then the inverse limit of the inverse system is a compact metric space homeomorphic to each $X_{m}$. Note that it follows that if each $X_{m}$ is $[0,1]$ and each $f_{m}$ is a homeomorphism from $[0,1]$ onto $[0,1]$, then the inverse limit of the inverse system is an arc.

Theorem 6 (Subcontinua) Suppose $\left(X_{m}, f_{m}\right)$ is an inverse limit sequence. If, for each $m, K_{m}$ is a subcontinuum of $X_{m}$ and $f_{m}\left(K_{m+1}\right)=K_{m}$, then $\lim _{(}\left(K_{m},\left.f_{m}\right|_{K_{m+1}}\right)$ is a subcontinuum of $\lim _{\longleftarrow}\left(X_{m}, f_{m}\right)$.

To repeat: If $X$ is a compact metric space, and $f$ is a continuous map from $X$ to $X$, then $\left(X_{m}, f_{m}\right)$, where $X_{m}=X$ and $f_{m}=f$ for each positive integer $m$, is an inverse system. With the simpler counting here, we can denote the corresponding inverse limit by $\lim (X, f)$ and not have problems with ambiguity. 
Let $X^{*}=\lim _{(}(X, f)$. In this case, a natural map is induced on the inverse limit space by the bonding map $f$ : for $\mathbf{x}=\left(x_{0}, x_{1}, \ldots\right) \in X^{*}$, define $f^{*}(\mathbf{x})=f^{*}\left(\left(x_{0}, x_{1}, \ldots\right)\right)=\left(f\left(x_{0}\right), f\left(x_{1}\right), \ldots\right)=\left(f\left(x_{0}\right), x_{0}, x_{1}, \ldots\right)$. The induced map $f^{*}$ is a homeomorphism from $X^{*}$ onto $X^{*}$. The inverse $g:=f^{*-1}$ of $f^{*}$ is then defined by $g(\mathbf{x})=g\left(\left(x_{0}, x_{1}, \ldots\right)\right)=\left(x_{1}, x_{2}, \ldots\right)$. Thus, the pair $\left(X^{*}, f^{*}\right)$ forms a dynamical system, one that runs both forward and backward. Both the induced map $f^{*}$ and its inverse are traditionally called the shift homeomorphisms. Note that we have, in a sense, "turned" a continuous map $f$ on a space $X$ into a homeomorphism $f^{*}$ on a possibly more complicated space $X^{*}$. Note also that the map $f$ on $X$ determines the inverse limit space itself. How complicated topologically the inverse limit space is, is a measure of the complexity of the dynamics of the original map on $X$. Because we are concerned here with the problem of backward dynamics, and $\left(f^{*}\right)^{-1}:=\sigma$ is the homeomorphism that takes us forward in time, we reserve the use of the word "shift" in this paper to mean $\left(f^{*}\right)^{-1}:=\sigma$.

A nice, well written introduction to inverse limits on an interval with one bonding map is given in [43], along with an investigation of the relationship between the complexity of the topology of the inverse limit space and the complexity of the dynamics on the resulting inverse space.

The theorems below are due to Tom Ingram.

Theorem 7 (|39|) Suppose $f$ is a Type (1) unimodal mapping of an interval $[a, b]$ with critical point $c$ onto itself, and $q$ is a point in $(c, b]$ such that $f^{2}(q)=q$ and $f(a)=q$. Then the inverse limit of the inverse limit system $([a, b], f)$ is the union of two Knaster bucket handle continua intersecting at a point or an arc.

Theorem 8 (|39|) Suppose $f$ is a Type (1) unimodal mapping of an interval $[a, b]$ onto itself and $q$ is the first fixed point for $f^{2}$ in $[c, b]$. Then $f$ has a periodic point of odd period greater than 1 if and only if $f^{2}(b)<q$.

Theorem 9 (|39|) Suppose $f$ is a Type (1) unimodal mapping of an interval $[a, b]$ onto itself and $q$ is the first fixed point for $f^{2}$ in $[c, b]$. Then $\lim _{\longleftarrow}([a, b], f)$ is indecomposable if and only if $f(a)<q$.

Theorem 10 (|39|) Suppose $f: X \rightarrow X$ and $g: X \rightarrow X$ are conjugate. Then their inverse limits $\lim _{(X}(X, f)$ and $\lim (X, g)$ are homeomorphic. Furthermore, if $F$ is the induced homeomorphism on $\underset{\lim }{\longleftarrow} \overleftarrow{(X}, f)$ and $G$ is the induced homeomorphism on $\lim _{\longleftarrow}(X, g)$, then $F$ and $G$ are conjugate, as are their respective shift maps.

Theorem $11(|39|)$ If I denotes an interval and $(I, f)$ is an inverse system such that $f$ is a homeomorphism, then the inverse limit of the inverse system is homeomorphic to an arc.

Theorem 12 (|40|) Suppose $f:[a, b] \rightarrow[a, b]$ is a continuous mapping, a is periodic of period $n \geq 3$ under $f$ and $b$ is in $O_{+}(a)$. If $k$ is an integer such that $f^{k}(a)$ is the first member of $O_{+}(a) \backslash\{a\}$, and $n$ and $k$ are relatively prime, then $\lim ([a, b], f)$ is an indecomposable continuum. (Note: $f^{k}(a)$ is the first member of $\mathrm{O}_{+}(a) \backslash\{a\}$ means first relative to the order on the interval $\left.[a, b].\right)$

Theorem 13 (|41|) Suppose $f:[a, b] \rightarrow[a, b]$ is a continuous mapping and is a Markov map with Markov partition $a=a_{1}<a_{2}<\cdots<a_{n}=b$ for $n \geq 3$ and $O_{+}(a)=\left\{a_{1}, a_{2}, \ldots, a_{n}\right\}$. If $k$ is an integer, $k<n$, such that $f^{k}(a)=a_{2}$, and $n$ and $k$ are relatively prime, then $\lim _{\longleftarrow}([a, b], f)$ is an arc continuum.

Barge and Martin [11] show that if the dynamics of $f$ are complicated, then the inverse limit contains indecomposable continua, and they give a partial converse under certain conditions on $f$.

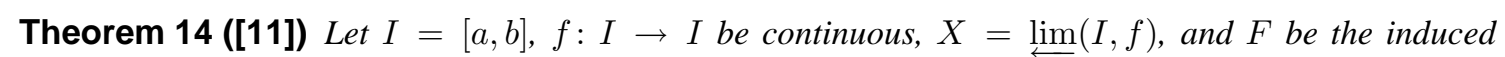
homeomorphism. Suppose $k$ and $n$ are integers with $k \geq 0, n \geq 1$, and that $f$ has a periodic point of power $2^{k}(2 n+1)$, i.e., not a power of 2 . Then $X$ contains an indecomposable continuum that is invariant under $F^{2^{k+1}}$.

Theorem 15 ([11|) Suppose $f: I \rightarrow I$ is continuous and onto with finitely many turning points. If

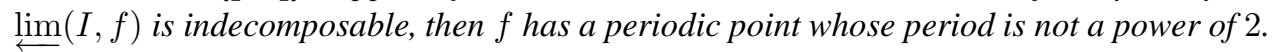




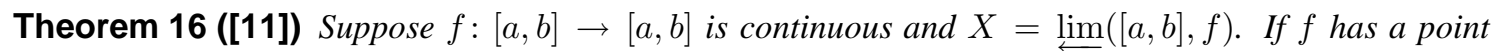
homoclinic to a periodic point then $X$ contains an indecomposable continuum.

Barge and Diamond [7] prove the following:

Theorem 17 (|7|) Suppose $f: X \rightarrow X$ is continuous with finitely many turning points, and $X$ is a finite graph (e.g., $X$ is an interval). Then the following are equivalent:

(a) The entropy $h(f)>0$.

(b) The inverse limit $\lim _{(}(X, f)$ contains an indecomposable continuum.

(c) The function $f$ has a horseshoe.

(d) There exist $r, M \in \mathbb{N}$ such that for $m \geq M$, $f$ has a periodic point of prime period $r m$.

\section{Results}

\subsection{CIA model-topology and dynamics}

In their paper [66], the economists Michener and Ravikumar noted the Li-Yorke paper [60], show that the map $f$ (from our discussion of the CIA model) can admit a period three point and also that it can admit a period 6 point, and conclude that whenever a period three point is present, this means the presence of chaos (in the sense of Li and Yorke). Michener and Ravikumar also cited the Sarkovskii Theorem and noted that presence of a period three point for $f$ meant that periodic points of all periods would be present. They essentially left it at that.

We note that while Kennedy and Yorke are mathematicians, Stockman is an economist. We can summarize our work in [54], [55], and [53] as follows: We show that the dynamical and topological behavior of the family of case I.A CIA maps we study is quite rich. As far as we can see now, it is just as rich as that of any of the extensively studied families of unimodal maps and inverse limits formed from those maps, although there is certainly work left to be done here, if one is to fully characterize this family. A result that economists found surprising was that the inverse limit space could be as simple as an arc, which would mean that the induced homeomorphism would be a homeomorphism on that arc, and that therefore, even though the map is not one-to-one, it would induce simple and completely understandable dynamics. One thing that bothered us about the Michener-Ravikumar paper was that [66] tried to understand the forward behavior of the system by looking exclusively at sequences of the form $\left(\ldots, x_{-2}, x_{-1}, x_{0}\right)$, that is, they looked backward in time (where the CIA map is well-defined). While one can obtain some information this way, such as, for example, knowledge of the periodic points, is looking at only the backward sequences going to fully reveal the properties of the system? In [53], we proved that, at least in the chaotic case, it essentially is $\mathrm{OK}$ to do this, i.e., to just study the backward sequences. We have also given a characterization of the utility function $U$ in terms of the interval map $f$. This makes it easy to use either the utility map $U$ or the backward map $f$ to produce examples and results. We provide more specifics below.

We focus on the interval $J=[\underline{x}, \bar{x}]$ (and case I.A) from now on, since any solutions that contain a point outside this interval behave in a simple way. Note that $\left.f\right|_{J}: J \rightarrow J$ is surjective. We denote $\left.f\right|_{J}$ as just $f$, since it should not lead to confusion.

If we consider the map $f: J \rightarrow J$ and form the inverse limit $X=\lim (J, f), X$ is a chainable continuum and can therefore be realized as a subset of the plane. The points of $X$ are precisely the solutions of the implicitly defined difference equation $A\left(x_{t+1}\right)=B\left(x_{t}\right)$ that stay in $J$. Denote by $F$ the homeomorphism induced on $X$ by $f$. Thus, for $x=\left(x_{0}, x_{1}, \ldots\right) \in X, F(x)=F\left(x_{0}, x_{1}, \ldots\right)=\left(f\left(x_{0}\right), f\left(x_{1}\right), f\left(x_{2}\right), \ldots\right)=$ $\left(f\left(x_{0}\right), x_{0}, x_{1}, \ldots\right)$. Let $\sigma=F^{-1}$, so that $\sigma$ is the shift map, and noting this, we denote $F^{-1}$ by $\sigma$.

The proofs can be found in [53], [54], and [55]. Since the proofs appear elsewhere, we do not include them here. Note that in the theorem below, we are back to representing $U$ as a function of $c_{1}$ and $c_{2}$. Under 
equilibrium conditions, it is the case that $c_{1 t}+c_{2 t}=y$ for all $t$ (that is, amount spent on cash goods plus amount spent on credit goods is the total endowment $y$ ), and it makes sense to write $U$ as a function of just one variable.

Theorem 18 ([55]) In the CIA model, $f$ is a type I.A backward map generated by a utility function $U\left(c_{1}, c_{2}\right)$ satisfying Assumption 1 (of [66]) with $0<\widetilde{\beta}<1$ if and only if $f$ is a type I.A map satisfying

1. for $x_{1} \geq x_{2}$, f is linear with slope $0<\widetilde{\beta}<1$ with $x_{2}=\bar{c} / \widetilde{\beta}$,

2. $f$ is $C^{1}$ on $[\underline{x}, \bar{x}] \backslash\left\{x_{1}, \bar{c}, x_{2}\right\}$, where $\underline{x}<x_{1}<\bar{c}$ is the unique solution to $f(x)=\bar{c}$,

3. for $x \in\left\{x_{1}, \bar{c}, x_{2}\right\}, f^{\prime}\left(x^{+}\right)$and $f^{\prime}\left(x^{-}\right)$exist, are non-zero with

$$
\frac{f^{\prime}\left(x_{1}^{-}\right)}{f^{\prime}\left(x_{1}^{+}\right)}=\frac{f^{\prime}\left(x_{2}^{+}\right)}{f^{\prime}\left(x_{2}^{-}\right)}, \quad \text { and }
$$

4. $x f^{\prime}(x)<f(x)$ for $x \in\left[\bar{c}, x_{2}\right]$.

In [53], we give meaning to $f^{-1}$ being chaotic, even though $f^{-1}$ is not a function. We also define what it means for $f^{-1}$ to have positive entropy. Those definitions are long, technical, and given in the inverse limit setting, and we do not include them here, but we note that it follows from results of Li [59] and Casanovas [21] that the induced homeomorphism $F$ and shift map $\sigma$ on the inverse limit space must be chaotic if and only if $f$ is chaotic, and $f$ has positive entropy if and only if $F$ and $\sigma$ have positive entropy.

Theorem 19 ([53|) Suppose $X$ is a compact metric space and $f: X \rightarrow X$ is continuous. Then $f^{-1}$ is chaotic (in the sense of Devaney) if and only if $f$ is chaotic on $X$.

Theorem 20 ([53|) Suppose $X$ is a compact metric space and $f: X \rightarrow X$ is continuous. Then $h(f)=$ $h\left(f^{-1}\right)$. Thus, $f^{-1}$ has positive entropy if and only if $f$ has positive entropy.

Applying the results discussed in the previous section, we see that if $\{\underline{x}, \bar{x}, c\}$ forms a period three orbit for $f, X$ is an indecomposable continuum. Furthermore, it follows from Sarkovskii's Theorem that $f$ has periodic orbits of all periods; and therefore, $\sigma: X \rightarrow X$ admits periodic orbits of all periods. However, $f$ may be chaotic only on an invariant Cantor set contained in the interval, or it may have dense set of periodic points (and thus be chaotic on the entire interval), or perhaps it could be something in between. We show that the first two cases can occur.

Most likely $f$ is contracting on $[c, \bar{x}]$, and we make that assumption in our examples. If $f$ is expanding on $[\underline{x}, c]$, and that expansion is large enough to dominate the contraction on the rest of the interval, then $f^{2}$ is expanding on the entire interval, and it follows from results of Baldwin [4] that the set of periodic points in the interval is dense, and $f$ is conjugate to the piecewise unimodal map $G$ on $[0,1]$ such that $G(0)=1 / 2$, $G(1 / 2)=1$, and $G(1)=0$.

Example 1 ([54|, Indecomposable Inverse Limit with Period Three Point and Dense Set of Periodic Points) We give an example of a map $f$ in our allowed family of maps (for convenience we have shifted the interval from $[\underline{x}, \bar{x}]$ to $[0,1])$ such that 0 and 1 are part of a period three orbit for $f$, and $f$ has a dense set of periodic points. Define $f:[0,1] \rightarrow[0,1]$ as follows:

$$
f(x)= \begin{cases}\frac{-20}{9} x+1 & \text { if } 0 \leq x \leq 0.45 \\ \frac{9}{11} x-\frac{(0.45)^{2}}{.55} & \text { if } 0.45 \leq x \leq 1\end{cases}
$$

Then $c:=0.45,\left.f^{\prime}\right|_{[0,0.45]}=\frac{-20}{9},\left.f^{\prime}\right|_{[0.45,1]}=\frac{9}{11}$. Note that $f$ is continuous and $\{0,0.45,1\}$ is a period 3 point. Also, $f$ is conjugate to the piecewise unimodal map $G$ on $[0,1]$ such that $G(0)=1 / 2, G(1 / 2)=1$, and $G(1)=0$. 
Theorem 21 (|54|) Define $g: J \rightarrow J$ as follows: $g(\underline{x})=\bar{x}, g(\bar{x})=\frac{\underline{x}+\bar{x}}{2}, g\left(\frac{\underline{\underline{x}}+\bar{x}}{2}\right)=\underline{x}, g$ is decreasing and linear on $\left[\underline{x}, \frac{\underline{x}+\bar{x}}{2}\right]$ and $g$ is increasing and linear on $\left[\frac{\underline{x}+\bar{x}}{2}, \bar{x}\right]$. Let $c=\underline{\underline{x}+\bar{x}} . h: J \rightarrow J, \underline{x}<c<\bar{x}$, and $h(\underline{x})=\bar{x}, h(\bar{x})=c$, and $h(c)=\underline{x}$ (so that $\{\underline{x}, c, \bar{x}\}$ forms a period three orbit in K). Furthermore, suppose $h$ is strictly decreasing on $[\underline{x}, c]$ and strictly increasing on $[c, \bar{x}]$, and $h^{2}$ is expanding on $[\underline{x}, \bar{x}]$. Then $h$ is weakly expanding on $[\underline{x}, \bar{x}]$. It follows that $h$ is weakly expanding on $K, h$ is conjugate to $g$, and we may completely understand the dynamics of $h$ on $K$ by considering the simpler piecewise linear map $g$. Furthermore, the orbit of some point in $K$ (under the action of $h$ ) is dense, and it must be the case that

1. there is a residual set of points in $K$ each of which has an orbit dense in $K$,

2. the set of periodic points in $K$ is dense in $K$, and

3. $h$ is sensitive to initial conditions at each point of $K$

Hence, $h$ is chaotic in the sense of Devaney on $K$. Then if $Y=\lim (K, h), Y$ is an indecomposable continuum and it is also an arc continuum. (Thus $Y$ is an indecomposable continuum, but it is rather simple for this class of continua in that it contains no indecomposable proper subcontinua.) Let $H$ denote the homeomorphism induced by $h$ on $Y$, and let $\sigma=H^{-1}$. It follows immediately that, under the action of $\sigma$, the set of points in $Y$ that have dense orbits in $Y$ is a residual subset of $Y$, the set of periodic points in $Y$ form a dense subset of $Y$, and $\sigma$ is sensitive to initial conditions in $Y$. In other words, $\sigma$ is chaotic in the sense of Devaney on $Y$.

It is not possible to picture completely an indecomposable continuum, and it is very difficult even to picture pieces of more than one composant. The application of an algorithm developed by Beverly Diamond and Karen Brucks [6] makes it possible to draw, with the help of a computer, accurate pictures of the developing continuum (or at least one composant of such a continuum) for an inverse limit space on an interval with one bonding map. For a nice picture of this "period three" continuum, see Nadler's Continuum Theory [69 Fig. 1.10, page 8].

Now suppose that the expansion on $[\underline{x}, c)$ does not dominate the contraction on $(c, \bar{x}]$ : Suppose there is an interval $L=[\underline{x}, d]$ in $[\underline{x}, c)$ such that $h^{3}(L) \subset[\underline{x}, d)$. (This might happen if, say, the max $\bar{x}$ of $h$ corresponds to the local maximum value of the original (case IA) $h$ defined on $[\underline{x}, c)$.) In this case the period three orbit $\{\underline{x}, \bar{x}, c\}$ is attracting for an open set of points in the interval:

Theorem 22 ([54]) Suppose $f:[\underline{x}, \bar{x}] \rightarrow[\underline{x}, \bar{x}]$ is continuous and has the following properties:

1. $f(\underline{x})=\bar{x}, f(\bar{x})=c($ where $\underline{x}<c<\bar{x})$, and $f(c)=\underline{x}$;

2. there is $d \in(\underline{x}, c)$ such that $\left.f\right|_{[\underline{x}, d]}$ is linear with negative slope $-m_{1}$ and $0<m_{1}<1$;

3. $\left.f\right|_{[d, c]}$ is linear with negative slope $-m_{2}$;

4. $\left.f\right|_{[c, \bar{x}]}$ is linear with positive slope $m_{3}<1$; and

5. $m_{2} m_{3}>1$ and $m_{1} m_{2} m_{3}<1$.

Then $\{\underline{x}, c, \bar{x}\}$ is an attracting period 3 point for $h$ and the basin $B$ of attraction of $\{\underline{x}, c, \bar{x}\}$ is dense in the interval $[\underline{x}, \bar{x}]$ and contains $[\underline{x}, d]$. Furthermore, $C:=[\underline{x}, \bar{x}] \backslash B$ is a Cantor set, $f(C)=C$, and $C$ contains periodic points of all periods.

Example 2 ([54], Indecomposable Inverse Limit with Period Three Attracting Orbit) The theorem above is not vacuous, i.e., such a map $f$ exists in our family of maps. Suppose $\underline{x}=0$ and $\bar{x}=1$ (we can translate later to change the interval without changing the dynamics). Define $f:[0,1] \rightarrow[0,1]$ as follows:

$$
f(x)= \begin{cases}1-(0.1) x, & \text { if } 0 \leq x \leq 0.1 \\ \frac{-99}{35} x+\frac{99(0.45)}{35} & \text { if } 0.1 \leq x \leq 0.45 \\ \frac{9}{11} x-\frac{(0.45)^{2}}{.55} & \text { if } 0.45 \leq x \leq 1\end{cases}
$$


Then $d=0.1, c=0.45, m_{1}=0.1, m_{2}=99 / 35, m_{3}=9 / 11$. Note that $f$ is continuous and $\{0,0.45,1\}$ is a period 3 point. Also, $m_{2} m_{3}=81 / 35>1$ and $m_{1} m_{2} m_{3}=81 / 350<1$.

Corollary 1 ([54]) Suppose $f:[\underline{x}, \bar{x}] \rightarrow[\underline{x}, \bar{x}]$ is continuous and satisfies the properties of the previous theorem. (The notation used for the theorem also applies here.) Then there is a continuous map $h:[\underline{x}, \bar{x}] \rightarrow$ $[\underline{x}, \bar{x}]$ with the following properties:

1. $h(\underline{x})=\bar{x}, h(\bar{x})=c($ where $\underline{x}<c<\bar{x})$, and $h(c)=\underline{x}$;

2. $h(x)=f(x)$ for $x \in[d, \bar{x}]$;

3. $h([\underline{x}, d])=f([\underline{x}, d])$; and

4. $\left.h\right|_{[\underline{x}, c)}$ is smooth and strictly decreasing.

Then $\{\underline{x}, c, \bar{x}\}$ is an attracting period 3 point and the basin $B_{h}$ of attraction of $\{\underline{x}, c, \bar{x}\}$ is dense in the interval $[\underline{x}, \bar{x}]$ and contains $[\underline{x}, d]$. Furthermore, $C_{h}:=[\underline{x}, \bar{x}] \backslash B_{h}$ is a Cantor set, $h\left(C_{h}\right)=C_{h}$, and $C_{h}$ contains periodic points of all periods.

Requiring $\left.h\right|_{[d, c]}$ to be linear seems to be a strong requirement, but it could undoubtedly be weakened and the result would still hold.

Next we show that the two Knaster bucket handle case can occur:

Example 3 ([55], Two Knaster Bucket Handle Inverse Limit ) Let $f: I \rightarrow I$ where

$$
f(x)= \begin{cases}m_{1}+m_{2} x & \text { if } x \in[a, d] \\ n_{1}+n_{2} x & \text { if } x \in[d, \bar{c}], \\ p_{1}+p_{2} x & \text { if } x \in[\bar{c}, b]\end{cases}
$$

with $I=[a, b], a=1, b=2, \bar{c}=a+(2 / 7)(b-a), d=(2 / 3) a+(1 / 3) \bar{c}, n_{2}=-1, m_{2}=-(b-a-$ $\bar{c}+d) /(d-a), m_{1}=b-m_{2} a, n_{1}=a-n_{2} \bar{c}, p_{2}=a /(\bar{c}+24 / 5), p_{1}=(24 / 5) p_{2}$. This map satisfies Ingram's Theorem 9 so that the resulting inverse limit space is two Knaster bucket handles joined at a point or an arc. Since $\lim (I, f)$ contains an indecomposable continuum, by Barge and Diamond's Theorem 19, $f$ must have positive topological entropy and $f$ contains a horseshoe.

Figure 7 illustrates a map on an interval conjugate to a member of our family that has simple dynamics. (It has been flipped and translated to the unit interval.) The inverse limit space is an arc or a double topologist's $\sin (1 / x)$ curve in this case (which of these we get depends on how $[0, e]$ and $[d, 1]$ interact for the map under the action of $f$ ), and the dynamics are therefore simple, even though forward in time the corresponding map from our family is not well defined. (See [43] for a picture of the double topologist's $\sin (1 / x)$ curve.)

Theorem 23 ([54]) Suppose $0<e<c<a<b<d<1$, and suppose $f:[0,1] \rightarrow[0,1]$ has the following properties:

(a) $f([a, b])=[e, d]$,

(b) $\left.f\right|_{[c, 1]}:[c, 1] \rightarrow[0,1]$ is one-to-one, onto, and decreasing,

(c) $\left.f\right|_{[0, c]}$ is increasing, and

(d) $f(c)=1, f(0)=d=f(a), f(b)=e, f(1)=0$.

Then $\varliminf_{(}([0,1], f)$ is either an arc or a double topologist's $\sin (1 / x)$ curve. 


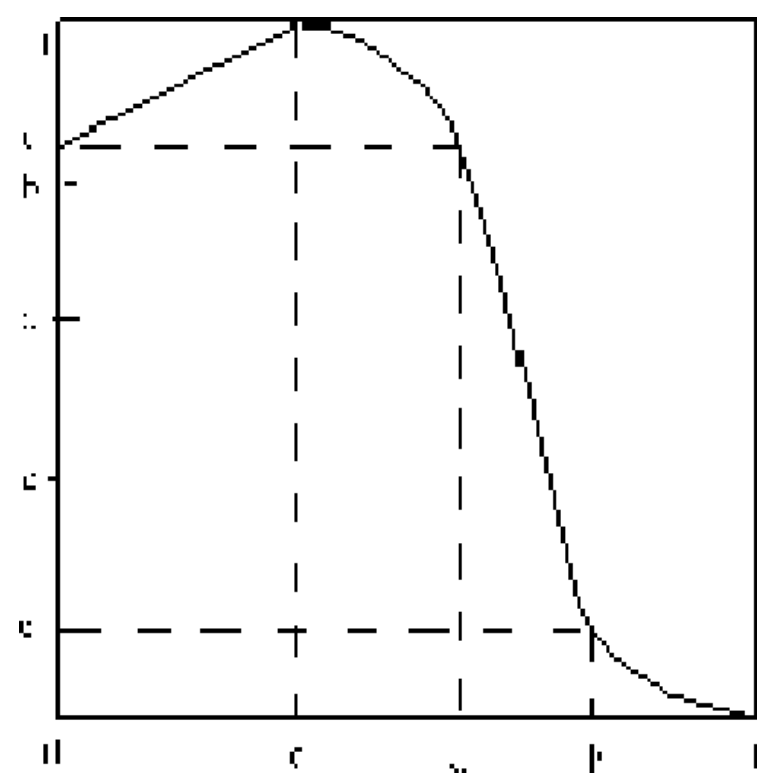

Figure 7.

Example 4 (|54|, Inverse Limit an arc) A map $f$ satisfying the hypotheses of Theorem 23 exists such that the inverse limit space $Z$ is an arc. The map is also conjugate to a member of our cash-in-advance family of maps: Suppose $e=0.2, c=0.6, a=0.7, b=0.8$, and $d=0.9$. There is a function $g:[0.7,0.8] \rightarrow[0.2,0.9]$ such that (i) $g^{\prime}(0.7)=g^{\prime}(0.8)=-1$, (ii) $g^{\prime}(x)<0$ for $x \in(0.7,0.8)$, and (iii) $g(0.7)=0.9, g(0.8)=0.2$. Define $f:[0,1] \rightarrow[0,1]$ as follows:

$$
f(x)= \begin{cases}\frac{x}{6}+0.9, & \text { if } 0 \leq x \leq 0.6 \\ -x+1.6, & \text { if } 0.6 \leq x \leq 0.7 \\ g(x) & \text { if } 0.7 \leq x \leq 0.8 \\ -x+1 & \text { if } 0.8 \leq x \leq 1\end{cases}
$$

Then $f$ is continuous on $[0,1]$ and satisfies the hypotheses of Theorem 23 Note that $f^{2}([0,0.2])=$ $f([0.9,28 / 30])=[1 / 15,0.1] \subset[0,0.2]$ and $f^{2}(x)=f(x / 6+0.9)=0.1-x / 6$ for $x \in[0,0.2]$. Also, $f^{2}([0.9,1])=f([0,0.1])=[0.9,55 / 60] \subset[0.9,1]$ and $f^{2}(x)=f(1-x)=-x / 6+64 / 60$ for $x \in[0.9,1]$. Hence, $I_{1}:=\cap_{n=0}^{\infty} f^{2 n}([0,0.2])$ consists of one point, as does $I_{2}:=\cap_{n=0}^{\infty} f^{2 n}([0.9,1]) \neq \varnothing$.

Example 5 (|54|, Inverse Limit a Double Topologist's $\operatorname{Sin}(1 / x)$ ) A map $f$ satisfying the hypotheses of Theorem 23 exists such that the inverse limit space $Z$ is a double topologist's $\sin (1 / x)$ curve. The map is also conjugate to a member of our cash-in-advance family of maps: Suppose $e=0.4, c=0.5, a=0.6$, $b=0.7$, and $d=0.8$. There is a function $g_{1}:[0.5,0.85] \rightarrow[0.275,1]$ such that (i) $g_{1}$ is continuously differentiable on $[0.5,0.85]$, (ii) $g_{1}^{\prime}(x)<0$ for $x \in[0.5,0.85]$, (iii) $g_{1}(0.5)=1, g_{1}(0.6)=0.8, g_{1}(0.7)=0.4$, $g_{1}(0.85)=0.275$, and (iv) $g_{1}^{\prime}(0.85)=-2.5$. There is a function $g_{2}:[0.95,1] \rightarrow[0,0.025]$ such that (i) $g_{2}$ is continuously differentiable on $[0.95,1]$, (ii) $g_{2}^{\prime}(x)<0$ for $x \in[0.95,1]$, (iii) $g_{2}(0.95)=.025, g_{2}(1)=0$, and (iv) $g_{2}^{\prime}(0.95)=-2.5$. Define $f:[0,1] \rightarrow[0,1]$ as follows:

$$
f(x)= \begin{cases}0.4 x+0.8, & \text { if } 0 \leq x \leq 0.5 \\ g_{1}(x) & \text { if } 0.5 \leq x \leq 0.85 \\ -\frac{5}{2} x+2.4 & \text { if } 0.85 \leq x \leq 0.95 \\ g_{2}(x) & \text { if } 0.95 \leq x \leq 1\end{cases}
$$


Then $f$ is continuous on $[0,1]$ and satisfies the hypotheses of Theorem 23 Note that $f^{2}(0.85)=0.91$ and $f^{2}(0.91)=0.85$. (In fact, each point in the interval $[0.85,0.91]$ is a period two point for $f^{2}$.) Thus, $[0.85,0.91] \subset \cap_{n=0}^{\infty} f^{2 n}([0.85,0.95])$ and $I_{2}$ is an interval in this case. Hence, $I_{1}:=\cap_{n=0}^{\infty} f^{2 n}([0,0.2])$ must be an interval, too. Then the inverse limit space for this example is a double topologist's $\sin (1 / x)$ curve.

We have shown that the inverse limit spaces from different members of our family of maps on an interval can behave quite differently, both topologically and dynamically. There are many more possibilities and we conjecture, although much remains to be done here, that all possibilities which follow from the body of tools for inverse limits developed in the last fifty or so years can occur:

1. Tom Ingram's theorems (Theorems 9 13) can all be satisfied for maps conjugate to many members of the family of maps for our cash-in-advance model. Thus, the resulting inverse limit can be an indecomposable continuum whether or not there are certain period points (Theorem 11] is satisfied).

2. If the map is a Markov map, then Ingram's Theorem 13 gives sufficient conditions under which the resulting inverse limit space is an arc continuum.

3. In [8], the authors proved that for a certain family $\left\{f_{\lambda}\right\}$ of tent maps and a residual set of parameters $(\lambda \in[1,2])$, the inverse limit space $\lim _{(}\left([0,1], f_{\lambda}\right)$ is not only indecomposable, it also contains homeomorphic copies of every inverse limit of a tent map $T_{\beta}$ for each $\beta \in[1,2]$. (The collection $\left\{T_{\beta}\right\}$ for $\beta \in[1,2]$ is another family of tent maps on $[0,1]$.) Thus, not only is this space complex, it has "sublayers" that are themselves complex. This continuum must contain uncountably many topologically different indecomposable proper subcontinua. We don't know if this is true for at least some member of our family of models, but we conjecture that it is.

There are many more questions one could ask, even about members of the family of maps we have studied, not to mention cases II.A and II.C (which we did not consider at all).

What does all this mean to an economist? What does the presence of chaos itself mean to an economist? The presence of chaos is interesting because it offers an alternative way of modeling fluctuations. The standard method of modeling fluctuations in economics is to use a random (stochastic) dynamical system where the fluctuations are due to exogenous random "shocks" to the system. However, a chaotic system allows for (deterministic) endogenous fluctuations without the need to introduce exogenous randomness to the model. Economists are also interested in knowing under what parametrization of the model is chaos possible. This potentially allows one to make policy prescription (e.g., in the CIA model, the growth rate of the money supply $\theta$ ) to eliminate the possibility of chaos (assuming this would be consistent with the central bank's goals). The fact that the inverse limit tells us something about the underlying dynamics is important to an economist because it offers a new way to explore and detect complicated dynamics. For example, the fact that $X$ can turn out to be an arc, and $F$ a homeomorphism from an arc to itself, so that the dynamics of the system are extremely simple, is significant to economists. It says that even though $f$ on $J$ is not one-to-one, and the corresponding difference equation is not well-defined forward in time, the behavior of the system can be predictable and simple rather than complicated and chaotic.

\subsection{The OLG model-topological attractors}

In [63] and [64], Medio (economist) and Raines (topologist) study the qualitative behavior of an OLG model of the 'Samuelson' type. As with the CIA model studied by Kennedy, Stockman, and Yorke, the model has the problem of backward dynamics. Medio and Raines identify three canonical types of maps coming from this OLG model, and describe the inverse limit space corresponding to each of them as well as the attractor of the associated shift map. The goal of the papers is to understand the structure of attractors (under the shift maps) that arise, because the forward admissible sequences that make up the attractors (metric or topological) are the ones that can be 'seen', and thus are the ones the implicitly defined system predicts. Again, since the proofs appear elsewhere, we give only most of the main results. 
Theorem $24(|64|)$ Let $f:[0,1] \longrightarrow[0,1]$ be unimodal. Let $\hat{A}$ be a closed, $\sigma$-invariant subset of $\underset{\lim }{\longleftarrow}([0,1], f)$ with $\pi_{1}(\hat{A})=A$. Suppose that $f^{-1}(A) \neq A$. Then $\hat{A}$ is not Liapunov stable, and there$\longleftarrow$ fore $\hat{A}$ is not asymptotically stable.

Corollary 2 (|64|) Let $f:[0,1] \rightarrow[0,1]$ be unimodal. Let $A$ be a periodic orbit under $f$ of period $n \geq 3$. Then $A$ is a closed invariant subset of $[0,1]$ under $f$, and we define $\lim _{(}\left(A,\left.f\right|_{A}\right)$. Then $\hat{A}$ is a closed, $\sigma$-invariant subset of $\lim _{(}([0,1], f), \hat{A}$ is not Liapunov stable, and therefore $\hat{A}$ is not asymptotically stable.

Medio-Raines then move on to consider unimodal maps of type A, type B, and type $\mathrm{C}$ (since interval maps coming from the OLG family can be any of these). They do not consider in detail the case in which $f(c)<c$ (where $c$ is the turning point for the unimodal map $f$ ), since in this case the inverse limit space $\underset{\lim }{\longleftarrow}([0,1], f)$ is simply an arc, and so the action of $\sigma$ on $\underset{\longleftarrow}{\longleftarrow}([0,1], f)$ is just the action of a homeomorphism on an arc.

Theorem 25 (|64|) Let $f$ be a type A unimodal map on $[0,1]$ with turning point $c$, and $\hat{0}=(0,0, \ldots)$. If $f^{2}(c)=f(1)>0$ and $f^{\prime}(0)>1$, then $\hat{0}$ is an asymptotically stable attractor. Moreover, if $f(x)>x$ for all $x \in(0, c)$, then $\hat{0}$ is the only topological attractor for $\sigma$ on $\underset{\lim }{\longleftarrow}([0,1], f)$.

If instead, $f^{2}(c)=f(1)=0$, then the usual unimodal maps (such as the tent or quadratic maps) on $[0,1]$ are transitive and so contain no topological attractors for $\sigma$. The only topological attractor in this case is the entire inverse limit space. For type B maps, the situation is not so simple.

Theorem $26(|64|)$ Let $f$ be a type $B$ unimodal map on $[0,1]$ with unique fixed point $p \in[c, 1]$, that is repelling on $[c, 1]$, and suppose $f(0)>p$. (Again, $c$ is the turning point for $f$.) Then the point $\hat{p}=$ $(p, p, \ldots) \in \lim ([0,1], f)$ is an asymptotically stable attractor, and it is the only topological attractor in $\underset{\lim }{\longleftarrow}([0,1], f)$.

It is possible to have a type B map with $f(0)>p$ and $p$ not repelling on $[c, 1]$. It could even be possible for $f$ to have a period two orbit contained in $[c, 1]$, that is repelling: this would generate a two-point set in $\lim ([0,1], f)$ that is an asymptotically stable attractor.

Next suppose $f$ is a type B unimodal map with $f(0) \leq p$. Now we have an incredibly rich family of maps, and they generate many types of inverse limit spaces. All must contain indecomposable continua and if $f(0)<p$, then the inverse limit space itself is an indecomposable continuum. Suppose then that we restrict ourselves to the case where $f$ is a type $\mathrm{B}$ unimodal map and $f(0)<p$, and we further assume that $f$ has a stable attractor. (This occurs for many unimodal maps with negative Schwarzian derivative. See [44].) For such type B unimodal maps with stable attractor $P$, then from results in [44], there are $n$ open intervals $A_{0}, A_{1}, \ldots, A_{n-1}$ such that $f^{i}\left(A_{0}\right) \subseteq A_{i}$ and $\cup_{i \in 0}^{n-1} A_{i}$ is the stable manifold of $P$. Then $f\left(\cup_{i \in 0}^{n-1} A_{i}\right) \subseteq \cup_{i \in 0}^{n-1} A_{i}$. Let $\Lambda=\left\{x \in[0,1]: f^{n}(x) \notin \cup_{i \in 0}^{n-1} A_{i}\right\}$. The restriction of $f$ to $\Lambda$ is conjugate to a subshift of finite type $\sigma_{A}$ on some sequence space $\sum_{A}$, and there is a decomposition of $\Lambda$ into a disjoint union of Cantor sets $\Lambda_{i}$ such that $\left.f\right|_{\Lambda_{i}}$ is topologically transitive. For simplicity, we assume that $\left.f\right|_{\Lambda}$ is topologically transitive. Let $\hat{\Lambda}=\lim (\Lambda, f \mid \Lambda)$.

Theorem 27 (|64|) Suppose $f$ is a type B unimodal map on $[0,1]$ with $f(0)<p$ (where $p$ is the unique fixed point for $f$ ), $f$ has a stable attractor $P$, and $f$ is topologically transitive on $\Lambda=\left\{x \in[0,1]: f^{n}(x) \notin\right.$ $\left.\cup_{i \in 0}^{n-1} A_{i}\right\}$ (with the sets $A_{i}$ defined as above). Then $\hat{\Lambda}=\lim \left(\Lambda,\left.f\right|_{\Lambda}\right)$ is a topological attractor for $\sigma$ on $\underset{\lim }{\longleftarrow}([0,1], f)$. Moreover, $\hat{\Lambda}$ is the only topological attractor.

Finally, consider the familiar logistic map $F_{\mu}(x)=\mu x(1-x)$ with $\mu>4$ playing the role of our 'canonical model' of a type $\mathrm{C}$ unimodal map. See Figure 8

Let $X_{1}=[0,1], X_{2}=X_{1} \cap F_{\mu}^{-1}\left(X_{1}\right)$, and inductively define $X_{i}=X_{i-1} \cap F_{\mu}^{-1}\left(X_{i-1}\right)$. Also define $f_{i}=\left.F_{\mu}\right|_{X_{i+1}}$, so that $f_{i}$ maps $X_{i+1}$ into $X_{i}$. Then $\left\{X_{i}, f_{i}\right\}$ is a inverse sequence and $\lim \left(X_{i}, f_{i}\right)$ is the associated inverse limit space (note that we have left the one bonding map situation) consisting of all forward admissible sequences permitted by the difference equation with $x_{i} \in[0,1]$. 


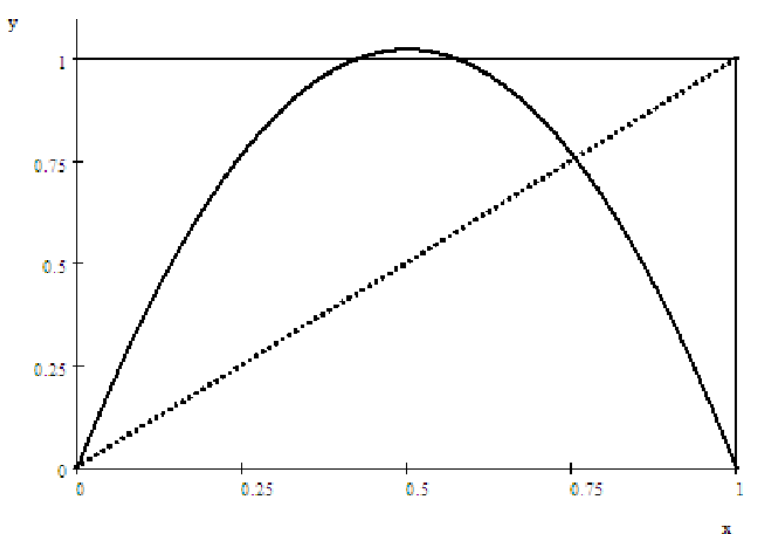

Figure 8. The logistic map $F_{\mu}$ with $\mu>4$.

In this case, $\lim _{(}\left(X_{i}, f_{i}\right)$ is homeomorphic to a product of a Cantor set and an arc [64]. Let $\sum_{2}$ denote the Cantor set $\left\{\left(z_{0}, z_{1}, \ldots\right): z_{i} \in\{0,1\}\right.$ for each nonnegative integer $\left.n\right\}$. Let $i$ denote the standard itinerary mapping defined by $i(x)=0$ if and only if $x<1 / 2$ and $i(x)=1$ otherwise for $x \in[0,1]$. Let $\Lambda=$ $\cap_{n \geq 0} F_{\mu}^{-1}([0,1])$, and let $\hat{\Lambda}=\varliminf_{\varlimsup}\left(\Lambda, F_{\mu}\right)$. Note that $\hat{\Lambda}$ is a homeomorphic embedding of $\Lambda$ in $\varliminf_{i}\left(X_{i}, f_{i}\right)$. Also, from [63], $\hat{\Lambda}$ is closed and a $\sigma$-invariant subset of $\varliminf_{\longleftarrow}\left(X_{i}, f_{i}\right)$.

Theorem 28 (|64|) Let $\mu>4$. Let $K \subseteq[0,1]$ such that $K$ is closed invariant and repelling under $F_{\mu}$. Let $\hat{K}=\lim \left(K,\left.F_{\mu}\right|_{K}\right)$. Then $\hat{K}$ is an attractor for $\lim \left(X_{i}, f_{i}\right)$ if and only if $K=\Lambda$, with $\Lambda$ defined as above. Furthermore, $\Lambda$ is homeomorphic to the product of a Cantor set and an arc, and it is closed and invariant under $\sigma$.

\subsection{Quantitative behavior}

While the work just described ([54, 55, 53, 63, 64]) gives a good start in applying inverse limits to the problem of backward dynamics, the results in those papers are qualitative and, while economists have an appreciation for qualitative results, they eventually like to get to quantitative results. So we felt we needed to move in that direction, and we have. We needed to "measure". Lebesgue measure just doesn't work on most inverse limit spaces. We needed to come up with a more appropriate and somehow "natural" measure.

We finally did that, and then discovered that it had almost been done long before (see [25]). But we had what we needed, a "natural" invariant measure on $\lim _{(i m}(J, f)$ for one of our backward maps $f$ defined on an interval $J$. While we applied all this to the type I. A CIA model example previously studied, our techniques can be applied much more broadly to dynamic equilibrium models (DGE's), and apply to direct limits (which also come up in many economic models) as well as inverse limits (which we have used to turn the original map $f$ taking us back in time, into a homeomorphism on the inverse limit space).

Specifically, suppose that an economic model's equilibria correspond to orbits generated by a chaotic dynamical system $f: X \rightarrow X$ where $X$ is a compact metric space and $f$ is continuous. The map $f$ could represent the forward dynamics $x_{t+1}=f\left(x_{t}\right)$ or the backward dynamics $x_{t}=f\left(x_{t+1}\right)$. If $f$ represents the forward/backward dynamics, the set of equilibria forms a direct/inverse limit space. We use an $f$-invariant measure on $X$ to induce a measure on the direct/inverse limit space and show that this induced measure is $\sigma$-invariant where $\sigma$ is the shift operator. Moreover, we show that if the $f$-invariant measure is a so-called natural invariant measure, then the induced measure on the direct/inverse limit space will also be a natural invariant measure. (Note: since Lebesgue measure makes no sense on many inverse limit spaces, we must give this term meaning. We cannot use the usual definition of natural measure, so we call these natural invariant inverse limit measures.) 
This section describes work of Kennedy-Raines-Stockman that appears in [51] and [52]. Since the direct limit results are quite easy to obtain and parallel the inverse limit results, and we haven't focused on forward dynamics anyway, we discuss only the inverse limit portion of the results. Again, we omit the proofs. At the end of this section, we give an example showing how these results can be applied to chaotic CIA maps and what it means for economics.

A content is a nonnegative, finite, monotone, additive, and subadditive set function defined on the class $\mathcal{C}$ of all compact sets of a locally compact metric space $X$. It is straightforward to generate a regular Borel measure from a content on the compact subsets of a locally compact space $X$, see [34, Section 53, p 231]. Thus, if we can define a content on $Y:=\lim (X, f)$, we can generate a measure on $Y$.

If $X$ is a metric space, $f: X \rightarrow X$ is continuous, and $\mu$ is a measure on $X$ with the property that $\mu\left[f^{-1}(S)\right]=\mu[S]$ for every closed set $S$ in $X$, then $\mu$ is called an invariant measure for $f$. Let $n$ be a nonnegative integer, and let $B$ be a compact subset of $Y$. Define the tower sets $B_{n}$ for $B$ as follows:

$$
B_{n}:=\left\{\mathbf{x} \in Y: \pi_{n}(\mathbf{x}):=x_{n} \in \pi_{n}(B)\right\} .
$$

Note that $\pi_{n}^{-1}\left(\pi_{n}(B)\right)=\pi_{n}^{-1}\left(\pi_{n}\left(B_{n}\right)\right)=B_{n}$, and that $B_{0} \supseteq B_{1} \supseteq \cdots \supseteq B$ and that $\cap_{n=0}^{\infty} B_{n}=B$.

Suppose that $\mu$ is an $f$-invariant measure on $X$. Now define the function $\Gamma$ on the compact subsets of $Y$ by first declaring that $\Gamma\left[B_{n}\right]=\mu\left[\pi_{n}(B)\right]$, where $B_{n}$ is a tower set for the compact set $B$ as defined above. Then define $\Gamma[B]=\lim _{n \rightarrow \infty} \Gamma\left[B_{n}\right]$. The function $\Gamma$ is a content on the compact sets of $Y$ :

Lemma 1 ([52|) The set function $\Gamma$ is a content on the compact sets of $Y:=\varliminf_{\longleftarrow}(X, f)$.

The content $\Gamma$ induces a Borel measure on $Y$, which we call $m$. We also have the following useful properties.

Lemma 2 ([52]) Let $K \subset Y$ be Borel. Then $m[K] \leq \mu\left[\pi_{n}(K)\right]$ for all nonnegative integers $n$ such that $\pi_{n}(K)$ is a Borel set in $X$.

Lemma 3 ([52|) Let $K \subset X$ be a Borel set. Then, for any nonnegative integer $n, \mu[K]=m\left[\pi_{n}^{-1}(K)\right]$.

A measure $\nu$ is nonatomic if $\nu(\{x\})=0$, for every $x \in X$. We call a measure strictly positive if it assigns every (nonempty) open set positive measure. The support of $\nu$ is the set of $x \in X$ such that every open set containing $x$ has positive measure. A measure $\nu$ has full support if the support of $\nu$ is all of $X$. Being a strictly positive measure is equivalent to having full support. Then we have the following result:

Theorem 29 ([52]) Suppose $X$ is a compact metric space, $f: X \rightarrow X$ is continuous, $Y:=\lim (X, f)$, $\mu$ is an invariant measure on $X$ with respect to $f$ such that $\mu$ is regular and nonatomic with $\mu(O)>0$ for each nonempty open set $O$ in $X$, and $m$ denotes the measure induced by $\mu$. Then the induced measure $m$ is $F$-invariant (and therefore $\sigma$-invariant).

Suppose we have a map $f: X \rightarrow X$ that is continuous and chaotic, with $X$ being a compact metric space. We would like to choose a measure $\mu$ on $X$ so that $\mu$ is not only invariant relative to $f$, but is also, in some sense "natural". After all, if $X$ is the unit interval $[0,1]$, then there is a fixed point $p$ for $f$. We could define $\mu[A]=1$ if $A \subset[0,1]$ and $p \in A$, and $\mu[A]=0$ if $A \subset[0,1]$ and $p \notin A$. Then $\mu$ is an invariant measure for $f$ on $[0,1]$, but what is it doing for us? It picks up the presence of the fixed point, and absolutely nothing else-including any chaos or total lack of it. It is not doing the job we want.

There is, fortunately, another approach - there is a large body of literature on what are called natural invariant measures (which are related SRB measures or Sinai-Ruelle-Bowen measures, SLYRB measures, and rain gauge measures). (See [1] for an introduction to these measures and [37] for a more in-depth discussion, survey, and reference list.) These measures address how the trajectory of a typical initial point is distributed asymptotically by the map $f$. What makes natural invariant measures so nice is that, when one exists, it really does measure the dynamics of $f$ in the sense that if almost all points have $60 \%$ of their respective orbits in $S$, then this measure assigns a value of 0.6 to $S$. Figuring out whether the natural invariant measure exists for a map $f$ can be difficult in general. 
Suppose $X$ is a compact metric space, $f: X \rightarrow X$ is continuous, $x_{0}$ is a point in $X$, and $S$ is a Borel subset of $X$. Define the fraction of the orbit of $x_{0}$ lying in $S$ by

$$
G_{f}\left(x_{0}, S\right)=\lim _{n \rightarrow \infty} \frac{\#\left\{f^{i}\left(x_{0}\right) \in S: 1 \leq i \leq n\right\}}{n},
$$

provided this limit exists.

Suppose $X$ is a compact metric space, $S$ is a subset of $X$, and $r$ is a positive number. Define $D_{r}(S)=$ $\{x \in X: d(x, y)<r$ for some $y \in S\}$. Note that $D_{r}(S)$ is an open set containing $S$ and as $r \rightarrow 0, D_{r}(S)$ shrinks down on $S$ (although $\cap_{r>0} D_{r}(S)$ may not be equal to $S$ ).

Suppose $X$ is a Euclidean space with Lebesgue measure $\lambda$. Let $f: X \rightarrow X$ be continuous, $x_{0}$ be a point in $X$, and $S$ be a compact subset of $X$. The natural measure generated by the map $f$ (or the $f$-measure) is defined by

$$
\mu_{f}(S)=\lim _{r \rightarrow 0} G_{f}\left(x_{0}, D_{r}(S)\right)
$$

provided that for $\lambda$-almost every $x_{0}$ this limit exists and is the same.

We would like to have a similar notion for an inverse limit space. However, if $f$ is chaotic, the inverse limit space is topologically complicated and is not a Euclidean space (or even close to it). Thus we give the following definition:

Suppose $X$ is a compact metric space, $f: X \rightarrow X$ is continuous, $Y:=\lim (X, f)$. If $\mathbf{x}_{0}$ is a point in $Y, S$ is a closed subset of $Y$, and $F$ is the induced homeomorphism on $Y$, then the natural inverse limit measure generated by $F$ is defined by

$$
\mu_{F}(S)=\lim _{r \rightarrow 0} G_{F}\left(x_{0}, D_{r}(S)\right)
$$

provided that for all $\mathbf{x}_{0} \notin Z$ this limit exists and is the same, where $Z$ is some set of the form $Z=\cup_{i=1}^{\infty} Z_{i}$ with $\lambda\left(\pi_{i}\left(Z_{i}\right)\right)=0$ for all $i \geq 0$.

There is at most one natural inverse limit measure for $F$. We have already used an invariant measure $\mu$ on $X$ to induce an invariant measure $m$ on $Y$. We can then show that the measure $m$ on the inverse limit space is the natural inverse limit measure induced by $F$ provided that $\mu$ is the natural measure induced by $f$.

Theorem 30 ([52]) Suppose $X$ is a compact metric space with Lebesgue measure $\lambda, f: X \rightarrow X$ is continuous, $Y:=\lim (X, f), \mu$ is a natural invariant measure on $X$ with respect to $f$ such that $\mu$ is nonatomic with full support, and $m$ denotes the measure induced by $\mu$. Then $m$ is a natural inverse limit measure on $Y$ for the induced homeomorphism $F: Y \rightarrow Y$, and $m$ is also a natural invariant inverse limit measure for $\sigma=F^{-1}$.

For $X$ compact and $f: X \rightarrow X$ continuous, once we have an invariant measure $\mu$ (natural or not) for $f$ on $X$, we therefore have an induced invariant measure $m$ on $Y:=\lim (X, f)$, and it is straightforward to show that we can then integrate on $Y$, that is, for $g: Y \rightarrow \mathbb{R}, \int_{Y} g d m$ makes sense.

Theorem 31 ([51]) Every continuous real-valued function on $Y$ is integrable relative to an induced natural invariant inverse limit measure.

From [51]: The framework here for calculating expected utility (which we do shortly) can be used to bridge two important literatures in macroeconomic theory: multiple equilibria and optimal policy. "Dynamic general equilibrium (DGE) models have become a standard framework for both the positive and normative evaluation of policy. In the optimal monetary/fiscal policy literature one considers a mapping from a policy space (e.g., money growth rate or set of taxes) to outcomes (e.g., allocations from a competitive equilibrium). If the mapping from policies to outcomes in the DGE model is single-valued, then one can induce a ranking on the policy space in a very natural way. For instance, suppose $\Theta$ is the policy space and for each $\theta \in \Theta$, there is a unique competitive equilibrium $E$ given by $E=M(\theta)$. If $U$ is the utility 


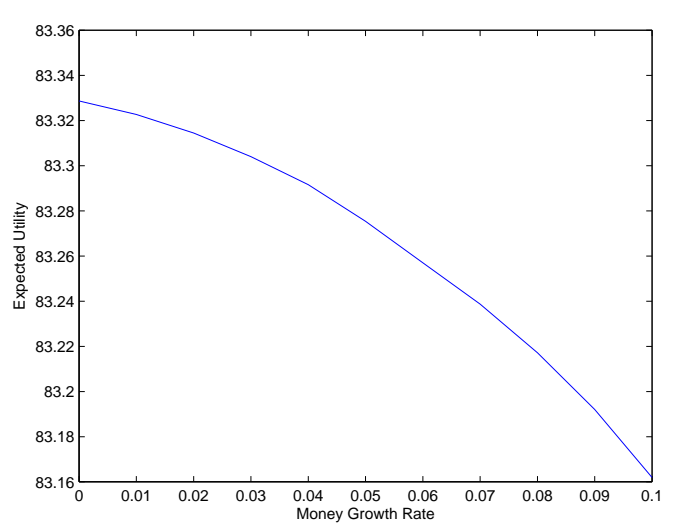

Figure 9.

function of the household defined over the space of competitive equilibria, then one can use the function $W(\theta):=U(M(\theta))$ to define a ranking on $\Theta$. In addition to perhaps locating the most preferred or optimal policy $\theta^{*}$, such a ranking can be used to measure the welfare gains of switching from some policy $\theta$ to another policy $\theta^{\prime}$. There is a large literature that takes this approach to evaluating policies starting with the work of Ramsey [74].

However, when $H$ is not single-valued this method of ranking policies will not work, and it is not clear what one should do since there is more than one equilibrium associated with a particular policy. There are many ways in which $M$ may be multi-valued. For example, the model may exhibit local indeterminacy in which for a given policy $\theta$ there exists a continuum of equilibria all converging to the steady state equilibrium. However, one may also have a multi-valued $H$ due to global properties of the model as well. Our framework can be applied to the class of economic models with equilibria that correspond to orbits generated by a chaotic dynamical system $f: X \rightarrow X$ where $X$ is a compact metric space and $f$ is continuous. Thus there are both a large number of equilibria and a large and complicated variety as well. Our framework is designed for this type of multi-valued $H$.

Finally, we move to an application to the CIA model [52]. A utility function $U$ (from [66]) that generates $f:[\underline{x}, \bar{x}] \rightarrow[\underline{x}, \bar{x}]$ is the following:

$$
U(x)=\frac{x^{1-\sigma}}{1-\sigma}+\frac{(y-x)^{1-\gamma}}{1-\gamma},
$$

where $\sigma>0$ and $\gamma>0$ and $y$ is the household's endowment. Then for $\mathbf{x}=\left(x_{0}, x_{1}, \ldots\right) \in Y$,

$$
W(\mathbf{x}):=\sum_{t=0}^{\infty} \beta^{t} U\left(x_{t}\right) .
$$

If we now compute

$$
\int_{X} W(\mathbf{x}) m(d \mathbf{x})
$$

we should obtain an expected utility. In one parameterization, [66 set $\beta=0.98, \sigma=0.5, \gamma=4.5$ and note that the CIA model exhibits chaos (the backward map has a three cycle) when money growth rates are $\theta$ equal to $0,0.5$ and 1.0. The backward map for this parameterization (with $\theta=0$ ) is pictured in Figure 9

To construct the natural $f$-invariant measure, we approximate $\mu$ via a histogram using a sample trajectory of $f$ for some $x \in[\underline{x}, \bar{x}]:\left\{x, f(x), f^{2}(x), \ldots\right\}$. This mimics the "rain gauge" description of the natural invariant measure described in [1]. Figure 10 contains an approximation of the density function for $\mu$. This histogram uses $10^{4}$ bins and a sample trajectory of length $10^{8}$. 


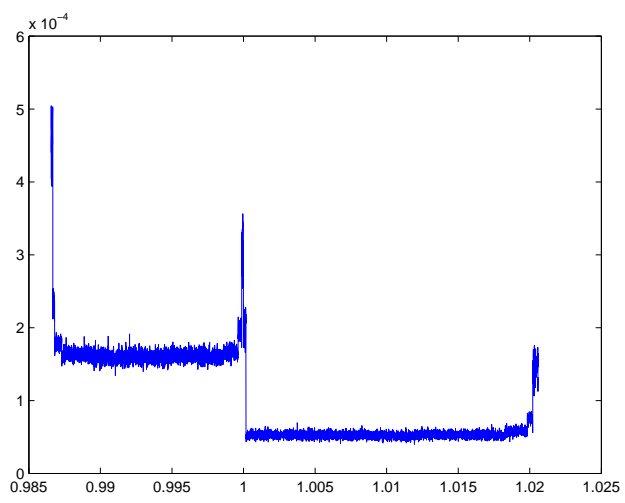

Figure 10.

Given this approximation to the natural invariant measure, the utility function $U$ and the discount factor $\beta$ it is now straight-forward to approximate our integral

$$
\int_{X} W(\mathbf{x}) m(d \mathbf{x})=\frac{1}{1-\beta} \int_{I} U(x) \mu(d x) \approx 83.3285573 .
$$

As mentioned in the introduction, our integral allows us to rank inverse limit spaces according to expected utility (a very natural ranking from the model). To give some sense of how this might be used to evaluate different monetary policies, imagine that for money growth rates $\theta \in \Theta:=[\underline{\theta}, \bar{\theta}]$, the backward map $f$ is chaotic. However, not all chaotic maps are the same in terms of utility. One way of framing the question through a Ramsey lens, is, within this subclass of possible monetary policies $\Theta$, which money growth rate gives the greatest expected utility? We see that for each $\theta \in \Theta$, we have a different backward map $f_{\theta}$, natural invariant measure $\mu_{\theta}$, invariant state space $I_{\theta}$, inverse limit space $X_{\theta}:=\lim \left(I_{\theta}, f_{\theta}\right)$, and induced natural inverse limit measure $m_{\theta}$. We then have an indirect utility function (or expected utility) given by

$$
V(\omega):=\int_{X_{\theta}} W(\mathbf{x}) m_{\theta}(d \mathbf{x}) \equiv \frac{1}{1-\beta} \int_{I_{\theta}} U(x) \mu_{\theta}(d x) .
$$

To be more concrete, suppose that the monetary authority is only considering money growth rates in $\Theta:=$ $[0,0.1]$. Which $\theta \in \Theta$ should the monetary authority choose to maximize expected utility? We see from Figure 9 that a lower money growth rate is preferred to higher money growth rate $\theta=0$ is the most preferred). This ranking is qualitatively similar to the ranking when considering only steady state equilibria. However, Figure 11 illustrates that considering only the steady state equilibria would underestimate the welfare costs of higher money growth rates.

Note that the framework given here is quite general and applies to any DGE model where the set of equilibria correspond to the orbits generated by a chaotic dynamical system $f: X \rightarrow X$ where $X$ is compact and $f$ is continuous with a natural invariant measure. In future research, we hope to extend our framework to DGE models with multiple equilibria where the underlying dynamical system does not admit a natural invariant measure. Such DGE models would include a large and important class of models, namely those with local indeterminacy."

\subsection{Another approach to measure}

In [65], Medio and Raines continue their find-the-attractors approach, but add a "Lebesgue-like" measure. If $X$ is a topological space and $\lambda: X \rightarrow \mathbb{R}$ is a measure on $X$, they define $\lambda$ as Lebesgue-like provided 


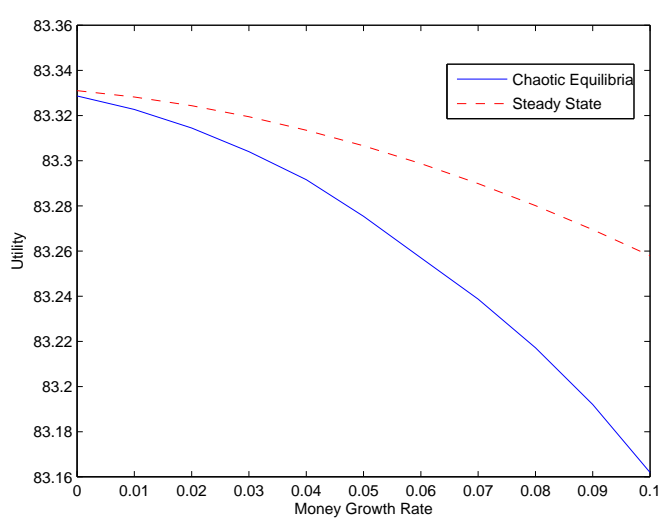

Figure 11.

1. $\lambda$ is a positive Borel measure,

2. if $U$ is open in $X$, then $\lambda(U)>0$, and

3. if $x \in X$, then $\lambda(\{x\})=0$.

They construct a Lebesgue-like measure for unimodal maps of the interval, and then use that measure to identify metric attractors (the previous work identified topological attractors) in the inverse limit for the shift homeomorphism. Thus, these results would then apply to the CIA and OLG models discussed here, as well as many others.

Their main results:

Theorem 32 Let $f:[0,1] \rightarrow[0,1]$ be a Type A unimodal map that has negative Schwarzian derivative such that $f(1)>0, f^{\prime}(x)>1$, for all $x \in\left[0, f^{2}\left(x^{*}\right)\right]$, where $x^{*} \in(0,1)$ is the turning point for $f$. Let $\hat{0}=(0,0, \ldots) \in \underset{\lim }{\longleftarrow}([0,1], f)$. Then $\{\hat{0}\}$ is the only metric attractor for $\lim _{\longleftarrow}([0,1], f)$ under $\sigma$.

Theorem 33 Let $f:[0,1] \rightarrow[0,1]$ be a transitive unimodal map that has negative Schwarzian derivative such that $f(1)=0$. Then the only attractor is the entire space $\lim ([0,1], f)$ (under $\sigma$ ).

Theorem 34 Let $f:[0,1] \rightarrow[0,1]$ be a Type B unimodal map that has negative Schwarzian derivative such that $f(0)>p$, where $p$ is the orientation reversing fixed point for $f$ in $\left[x^{*}, 1\right]$ (where $x^{*} \in(0,1)$ is the turning point for $f)$, and $\left|f^{\prime}(p)\right|>1$. Then $\lim _{(}([0,1], f)$.

Theorem 35 Let $f:[0,1] \rightarrow[0,1]$ be a Type B unimodal map that has negative Schwarzian derivative such that $f(0) \leq p$, where $p$ is fixed point for $f$ in $[0,1]$, and such that $f$ has a stable attractor which is either chaotic or periodic. Then $\hat{p}=(p, p, \ldots) \in \lim ([0,1], f)$ is the metric attractor for $\lim ([0,1], f)$. Then the metric attractor is the set $\hat{\Lambda}$. (For the definition of $\hat{\Lambda}$, see the previous section discussing Medio-Raines work.)

\subsection{And if a model is not well defined either backward or forward?}

There are a number of models coming from economics that are not well-defined either backward or forward in time. The Christiano-Harrison model (see [24]) pictured in Figure 12] is an example of such a model. While we do not go into the derivation of this model, we note that here the marvelous tools developed in the literature for inverse limits are not available. Topologists have recently begun studying these so-called "generalized" inverse limits, but this study is in its infancy and seems to be quite difficult and much more complicated than the usual inverse limit case. There is much for both mathematicians and economists to do in this situation. 


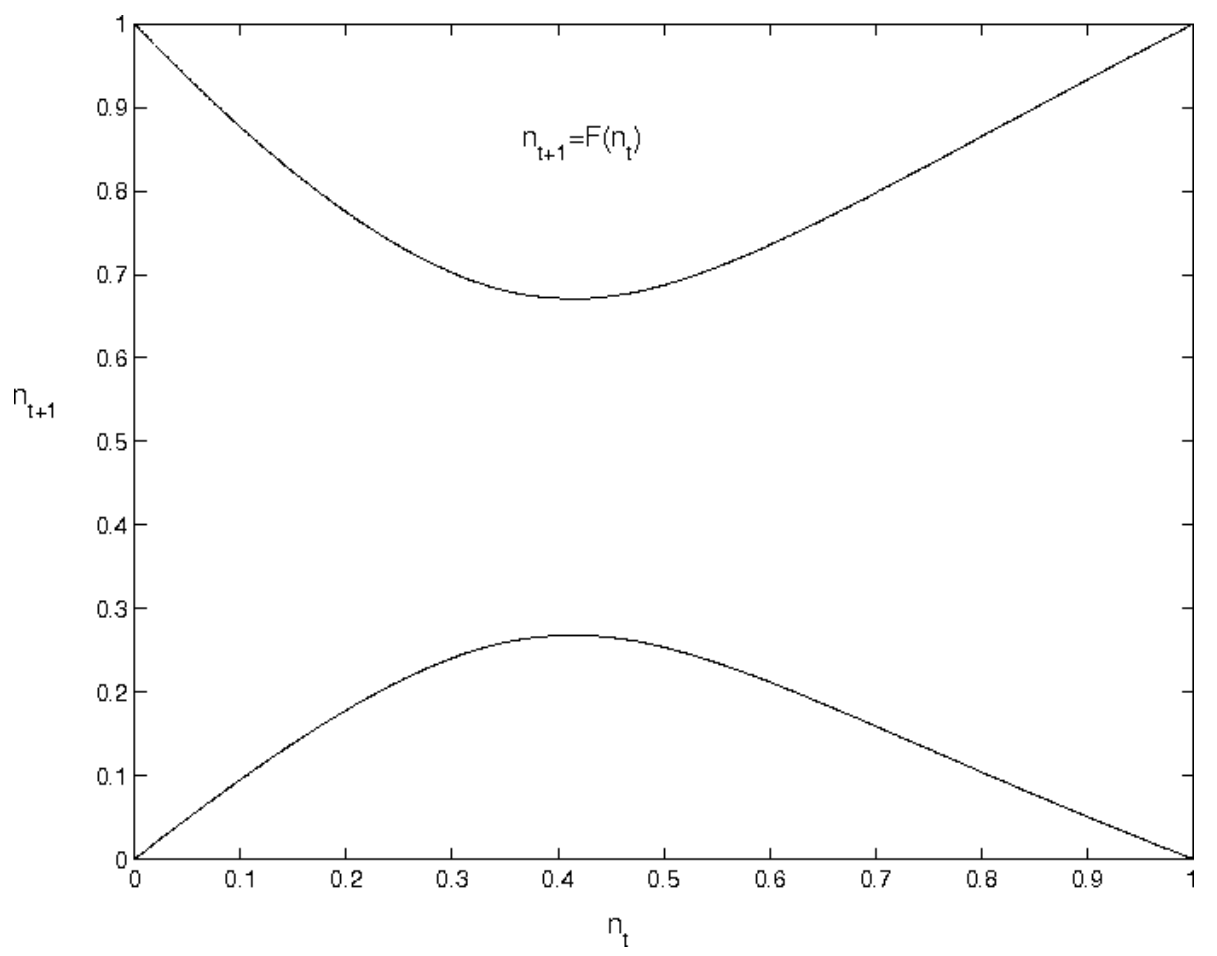

Figure 12.

\section{References}

[1] Alligood, K., Sauer, T. and Yorke, J. A., (1996). Chaos: An Introduction to Dynamical Systems, Springer, New York.

[2] Avila, A., Lyubich, M. and deMelo, W., (2004). Regular or stochastic dynamics in real analytic families of unimodal maps, Ann. Math., 156, 1-78.

[3] Azariadis, C., (1993). Intertemporal Macroeconomics, Blackwell, Cambridge MA,

[4] Baldwin, S., (1990). A complete classification of the piecewise monotone functions on the interval, Trans. Amer. Math. Soc., 319, 155-178.

[5] Banks, J., Brooks, J., Cairns, G., Davis, G. and Stacey, P., (1992). On Devaney's definition of chaos, Amer. Math. Monthly, 99, 332-334.

[6] Barge, M. and Diamond, B., (1998). Inverse limit spaces of infinitely renormalizable maps, Topology and its Applications, 83, 103-108.

[7] Barge, M. and Diamond, B., (1994). The dynamics of continuous maps of finite graphs through inverse limits, Trans. Amer. Math. Soc., 344, 773-790.

[8] Barge, M., Brucks, K. and Diamond, B., (1996). Self-similarity in inverse limit spaces of the tent family, Proc. Amer. Math. Soc., 124, 3563-3570.

[9] Barge, M. and Ingram, W. T., (1996). Inverse limits on [0,1] using logistic bonding maps, Topology and its Applications, 72, 159-172.

[10] Barge, M. and Martin, J., (1995). Endpoints of inverse limit spaces and dynamics, Continua (Cincinnati, OH, 1994), Lecture Notes in Pure and Appl. Math, 170, 165-182, Dekker, New York, . 


\section{J. Kennedy}

[11] Barge, M. and Martin, J., (1985). Chaos, periodicity, and snakelike continua, Trans. Amer. Math. Soc., 289, $355-365$.

[12] Benhabib, J. and Day, R., (1982). A characterization of erratic dynamics in the overlapping generations model, Journal of Economic Dynamics and Control, 4, 37-55.

[13] Blokh, A. and Lyubich, M. Yu., (1991). Measurable dynamics of S-unimodal maps of the interval, Ann. Sci. École Norm. Sup., 24, 545-573.

[14] Bochner, S., (1955). Harmonic Analysis and the theory of probability, University of California Press, Berkeley and Los Angeles.

[15] Boldrin, M. and Woodford, M., (1990). Equilibrium models displaying endogenous fluctuations and chaos: a survey, Journal of Monetary Economics, 25, 189-222.

[16] Bowen, R., (1975). A horseshoe with positive measure, Invent. Math., 29, $203-204$.

[17] Brucks, K. and Diamond, B., (1995). A symbolic representation of inverse limit spaces for a class of unimodal maps, Continua (Cincinnati, OH, 1994), Lecture Notes in Pure and Appl. Math 170, 207-226, Dekker, New York.

[18] Brucks, K. and Bruin, H., (1999). Subcontinua of inverse limit spaces of unimodal maps, Fund. Math., 160, 219-246.

[19] Bruin, H., (2000). Inverse limit spaces of post-critically finite tent maps, Fund. Math., 165, 125-138.

[20] Bruin, H., Keller, G., Nowicki, T. and van Strien, S., (1996). Wild Cantor attactors exist, Ann. of Math., 143, 97-130.

[21] Casanovas, J., (1999). On topological sequence entropy and chaotic maps on inverse limit spaces, Acta Math. Univ. Comenianae, 63, 205-211.

[22] Chari, V. and Kehoe, P., (1999). Optimal fiscal and monetary policy, in Handbook of Macroeconomics, J. Taylor and M. Woodford, eds., IC, North-Holland, New York.

[23] Chigogidze, A., (1996). Inverse Spectra, North-Holland Mathematical Library, vol. 53, North-Holland, Amsterdam.

[24] Christiano, L. and Harrison, S., (1999). Chaos, sunspots and automatic stabilizers, Journal of Monetary Economics, 44, 3-31.

[25] Chokski, J., (1958). Inverse limits of measure spaces, Proc. London Math. Soc., 8, 321-342.

[26] Devaney, R., (2003). An Introduction to Chaotic Dynamical Systems, second edition, Westview Press.

[27] Gale, D., (1973). Pure exchange equilibrium of dynamic economic models, Journal of Economic Theory, 6, $12-36$.

[28] Grandmont, J.-M., (1985). On endogenous competitive business cycles, Econometrica, 53, 995-1045.

[29] Grandmont, J.-M., (1983). On endogenous competitive business cycles, in Models of Economic Dynamics, H. F. Sonnenschein (ed.), Lecture Notes in Economics and Mathematical Systems Series, 264, Springer, New York.

[30] Grandmont, J.-M., (1989). Local bifurcations and stationary sunspots, in Economic Complexity: Chaos, Sunspots, Bubbles and Nonlinearity, W. A. Barnett, J. Geweke, K. Shell (eds.), Cambridge University Press, Cambridge, MA.

[31] Grandmont, J.-M., Kirman, A. P. and Neuefeind, W., (1974). A new approach to the uniqueness of equilibrium, Review of Economic Studies, 41, 289-291.

[32] Guckenheimer, J., (1979). Sensitive dependence to initial conditions for one-dimensional maps, Comm. Math. Phys., 70, 133-160. 
[33] Haller, H. and Lagunoff, R., (2000). Genericity and Markovian behavior in stochastic games, Econometrica, 68, 1231-1248.

[34] Halmos, P., (1958). Measure Theory, Springer-Verlag, New York.

[35] Hocking, J. G. and Young, G. S., (1998). Topology, 2nd ed., Dover, New York.

[36] Hommes, C. and deVilder, R., (1995). Sunspot equilibria in an implicity defined overlapping generations model, unpublished paper.

[37] Hunt, B., Kennedy, J., Li, T.-Y. and Nusse, H., (2002). SLYRB measures: natural invariant measures for chaotic systems, Physica D, 170, 50-71.

[38] Ingram, W. T., (2000). Inverse Limits, Aportaciones Matemáticas, Investigación 15, Sociedad Matemática Mexicana.

[39] Ingram, W. T., (1995). Periodicity and indecomposability, Proc. Amer. Math. Soc., 123, 1907-1916.

[40] Ingram, W. T., (2002). Invariant sets and inverse limits, Topology and its Applications, 126, 393-408.

[41] Ingram, W. T., (2003). Families of inverse limits, Topology Proceedings, 27, 189-201.

[42] Ingram, W. T., (2000). Inverse limits on [0, 1] using piecewise linear unimodal bonding maps, Proc. Amer. Math. Soc., 128, 279-286.

[43] Ingram, W. T. and Mahavier, W., (2004). Interesting dynamics and inverse limits in a family of one-dimensional maps, American Mathematical Monthly, 111, 198-215.

[44] Jonker, L. and Rand, D., (1981). Bifurcations in one dimension I: the nonwandering set, Invent. Math., 62, 347365 .

[45] Kailhofer, L., (2002). A partial classification of inverse limit spaces of tent maps with periodic critical points, Topology and its Applications, 123, 235-265.

[46] Kelley, J. L., (1975). General Topology, Springer, New York.

[47] Katok, A. and Hasselblatt, B., (1995). Introduction to the Modern Theory of Dynamical Systems, Cambridge University Press, New York.

[48] Kehoe, T. and Levine, D., (1985). Comparative statics and perfect foresight in infinite horizon economies, Econometrica, 53, 433-453.

[49] Kennedy, J., (1993). How indecomposable continua arise in dynamical systems, in Papers on General Topology and Applications, (Madison, WI, 1991), Annals of the New York Academy of Sciences, vol. 704, New York, 180-201.

[50] Kennedy, J., (1995) A brief history of indecomposable continua, in Continua (Cincinnati, OH, 1994), Lecture Notes in Pure and Applied Mathematics, vol. 170, Dekker, New York, 103-126.

[51] Kennedy, J., Raines, B. and Stockman, D. R., Expected utility in models with chaos, submitted.

[52] Kennedy, J., Raines, B. and Stockman, D. R. Natural invariant measures for the shift map on inverse limit spaces, preprint.

[53] Kennedy, J. and Stockman, D. R. Chaotic equilibria in models with backward dynamics, Journal of Economic Dynamics and Control, to appear.

[54] Kennedy, J., Stockman, D. R. and Yorke, J. A., (2007). Inverse limits and an implicitly defined difference equation from economics, Topology and its Applications, 154, 2533-2552.

[55] Kennedy, J., Stockman, D. R. and Yorke, J. A. Inverse limits and models with backward dynamics, Journal of Mathematical Economics, to appear. 


\section{J. Kennedy}

[56] Kirman, A., (1981). Measure theory with applications to economics, in Handbook of Mathematical Economics, K. Arrow and M. Intriligator (eds.), vol. I, North-Holland, Amsterdam, ,159-209.

[57] Kraft, R., (1999). Chaos, Cantor sets and hyperbolicity for logistic maps, Amer. Math. Monthly, 106, 400-408.

[58] Lucas, R. E. and Stokey, N. L., (1987). Money and interest in a cash-in-advance economy, Econometrica, 12, 491-514.

[59] Li, S., (1992). Dynamical properties of the shift maps on the inverse limit spaces, Ergodic Theory and Dynamical Systems, 12, 95-108.

[60] Li, T. Y. and Yorke, J. A., (1975). Period three implies chaos, American Mathematical Monthly, 82, 985-992.

[61] Medio, A., (1992). Chaotic Dynamics: Theory and Applications to Economics, Cambridge University Press, Cambridge.

[62] Medio, A., (1998). The problem of backward dynamics in economics, Working paper \#98.05, University Ca' Foscari of Venice.

[63] Medio, A. and Raines, B., (2007). Backward dynamics in economics: the inverse limit approach, Journal of Economic Dynamics and Control, 31, 1633-1671.

[64] Medio, A. and Raines, B., (2006). Inverse limit spaces arising from problems in economics, Topology and its Applications 153, 3437-3449.

[65] Medio, A. and Raines, B. A Lebesgue-like measure for inverse limit spaces that arise in economics, preprint.

[66] Michener, R. and Ravikumar, B., (1998). Chaotic dynamics in a cash-in-advance economy, Journal of Economic Dynamics and Control 22, 1117-1137.

[67] Milnor, J., (1985). On the concept of an attractor, Comm. Math. Phys., 99, 177-195.

[68] Munkres, J. R., (1975). Topology - A First Course, Prentice-Hall, Englewood Cliffs, NJ.

[69] Nadler, S., (1992). Continuum Theory - An Introduction Monographs and Textbooks in Pure and Applied Mathematics, 158, Marcel Dekker, New York.

[70] Oxtoby, J. C., (1971). Measure and Category, Springer, New York.

[71] Raines, B., (2002). Inverse limits on [0, 1] using sequences of piecewise linear unimodal bonding maps, Topology and its Applications, 124, 159-170.

[72] Raines, B., (2002). One-dimensional dynamics and inverse limits, D. Phil. thesis, Oxford University.

[73] Raines, B., (2004). Inhomogeneities in non-hyperbolic one-dimensional invariant sets, Fund. Math., 182, 241268.

[74] Ramsey, F., (1927). A contribution to the theory of taxation, The Economic Journal, 37, 47-61.

[75] Roe, R., (1993). Dynamics and indecomposable inverse limit spaces of maps on finite graphs, Topology and its Applications, 50, 117-128.

[76] Samuelson, P., (1958). An exact consumption-loan model of interest with or without the social contrivance of money, Journal of Political Economy, 66, 467-482.

[77] Vellekoop, M. and Berglund, R., (1994). On intervals: transitivity is chaos, Amer. Math. Monthly, 101, 353-355.

[78] deVilder, R. Endogenous business cycles, Tinbergen Institute Research Series n. 96.

[79] Walters, P., (1982). An Introduction to Ergodic Theory, Springer-Verlag, New York.

[80] Willard, S., (2004). General Topology, Dover, Mineola, NY. 
[81] Woodford, M., (1986). Stationary sunspot equilibria in a finance constrained economy, Journal of Economic Theory, 40, 128-137.

[82] Woodford, M., (1994). Monetary policy and price level determinacy in a cash-in-advance economy, Economic Theory, 4, 345-380.

\author{
Judy Kennedy \\ Department of Mathematics \\ Lamar University \\ P.O. Box 10047 \\ Beaumont TX 7710, and \\ Department of Math Sciences \\ University of Delaware \\ Newark DE 19711
}

